\title{
A Model of the Universe that Can Explain Dark Matter, Dark Energy, and the Fourth Space Dimension
}

\author{
Donald J. Koterwas ${ }^{1,2,3}$ \\ ${ }^{1}$ Retired, Monument, CO, USA \\ ${ }^{2}$ Department of Earth, Space, and Graphic Sciences, United States Military Academy, West Point, NY, USA \\ ${ }^{3}$ Department of Civil Engineering and Engineering Mechanics, and Department of Optics, University of Arizona, \\ Tucson, AZ, USA \\ Email:sdkoterwas@gmail.com
}

Received 29 April 2016; accepted 24 June 2016; published 28 June 2016

Copyright (C) 2016 by author and Scientific Research Publishing Inc.

This work is licensed under the Creative Commons Attribution International License (CC BY).

http://creativecommons.org/licenses/by/4.0/

(c) (i) Open Access

\section{Abstract}

This paper explains how a model of the universe can be constructed by incorporating time and space into geometry in a unique way to produce a 4-space dimension/1-time dimension model. The model can then show how dark matter can be the gravity that is produced by real matter that exists throughout our entire universe. The model can also show how dark energy is not an increase in energy that is causing the accelerated expansion of the universe, but is an accelerating decrease in matter throughout the universe as the stars and galaxies in the universe continue to convert matter into energy during their life cycles. And then the model can show how a fourth space dimension must exist in our universe to locate a point in space.

\section{Keywords}

Universe, Dark Matter, Dark Energy, Gravity, Fourth Space Dimension

\section{Introduction}

Dark matter and dark energy continue to be the subjects of many articles written in scientific papers and journals. Dark matter was proposed in the 1970's to account for the gravity that had to exist in a halo effect around galaxies to account for the faster than expected motion of stars in the outer orbits of the galaxies. It was called dark matter because it was not visible. It did not absorb, reflect, or emit light. It was estimated that the amount of dark matter had to be over 6 times the amount of visible matter that existed in the galaxies. Dark energy was pro- 
posed by astronomers in 1998 to account for the accelerating expansion of the universe that had become evident from their observations of the motions of supernovae and galaxies.

Most of the research that is done to try to explain dark matter involves the search for some kind of exotic particle that is not visible, but does produce a gravitational effect. There is an alternative way to try to explain dark matter. Dark matter does not have to be matter; it can just be gravity.

Dark energy is still a mystery. It is thought to be some kind of energy that is spread throughout the universe and is increasing at an accelerating rate. There is an alternative way to explain dark energy. The accelerating expansion of the universe does not have to be caused by an increase in energy that we call dark energy; it can be caused by a decrease in gravity due to a decrease in the amount of matter in the universe.

Both of these explanations involve gravity, and it is not surprising that gravity is still a little bit of a mystery. Is it a force that acts between objects as described by Sir Isaac Newton, or is it a warping of space-time as described by Albert Einstein? Regardless of its true nature, the effects of gravity in our universe are well known, and they can be shown most effectively by the use of equations, graphs, and geometric models. We can construct a model of the universe that will show the relationships between gravity, dark matter, and dark energy. The model will also show that a fourth space dimension must exist in our universe to locate a point in space.

\section{The Model}

A model of the universe can be constructed that can explain the true nature of dark matter and dark energy. Current models of the universe do not incorporate time into space in a way that shows the whole universe as it really exists on a grand scale. Some of the models show how the universe developed over time since the beginning of time at the big bang. Some show time as a timeline and show the development of the parts of the universe, such as protons, molecules, forces, stars, and galaxies, along this timeline. Some present a model in two or three dimensions upon which accurate measurements involving space and time can be made, such as the Lambda CDM cosmological model. But they do not present a 5-dimensional model of our entire universe upon which accurate measurements involving space and time can be made.

In our real world, we know that when we look out into space, we are also simultaneously looking back in time. And no matter in which direction we look, we are looking back in time about 13.8 billion years to the beginning of the universe at the big bang. We know that the Hubble telescope will give us a similar picture of the beginnings of our universe no matter in which direction it is pointed. It does not give us a picture of our nearest galaxies as they exist now. It gives us a picture of them as they existed millions and billions of years ago, since it has taken millions and billions of years for their emitted light to reach the telescope.

Time and space can be incorporated into geometry to construct a model that reflects the reality that the Hubble space telescope reveals. When mathematicians and scientists need to include time in their calculations about the physical world, they generally represent it as another dimension along a straight line equivalent to the $\mathrm{x}, \mathrm{y}$, or z-axis in the Cartesian coordinate system. Most of the current models of the universe also treat time as a straight line in one direction; i.e., as the arrow of time. However, if we consider the nature of time, we realize that it began at the creation of the universe, and it did not travel in a straight line in one direction in space. It progressed outward from the big bang in all directions. So, we can make a unique shift in incorporating time and space into geometry by treating time as a radius of a sphere in a coordinate system where time is the radius, and the three space coordinates, $\mathrm{x}, \mathrm{y}$, and $\mathrm{z}$ are on the surface of the sphere. As time proceeds forward and outward on the radii of the sphere, all matter in the universe moves outward and expands on the surface of the sphere. Measurements are made on this model by, first of all, placing the observer at a point in time on the radius, and then secondly, establishing his space coordinate system on the surface of the sphere which represents the universe, to make physical measurements in the usual manner. When time is involved in the measurement, the observer moves to the next point in time on the radius, and then on the surface of the sphere, which will now be a larger or smaller sphere (the universe), the space coordinate system is established to make the physical measurements.

We can visualize this model of the universe and how it works if we place some measurements and objects on it. Along the radii, time would be measured in years. Along the surface of the sphere, which represents all of space, distances are measured in light years. In this way, the model is scaled so that a unit of distance along the time line, say one inch representing one billion years, is equivalent to the same unit of distance along the surface of the sphere, which would be one inch representing one billion light years. This model is based on this equiva- 
lency, which recognizes the fact that the speed of light is one of the fundamental building blocks of our universe. When Albert Einstein developed his theory of relativity and produced his famous equation, $E=m c^{2}$, he showed how fundamentally important the speed of light is in our universe. It is not light itself, or electromagnetic waves in general, but it is the speed at which they travel through space that is so important. The speed of light is a limit to which nothing can travel faster. It actually presents a boundary to our universe. Speed is measured in units of distance divided by time, such as kilometers per second, or miles per hour. Thus the speed of light gives us a way of making measurements of distance, equivalent to measurements of time; i.e. one year of time is equivalent to one light year of distance in our universe. In our model, a length along the radius of the sphere, which represents time, is the same length along the radius that represents a measurement of one light year, and is also the same length along the surface of the sphere, which represents a distance in space. Because our model of the universe is based on this real equivalence, it will give us accurate measurements of space and time in our universe.

In our model, the beginning of time at $\mathrm{t}=0$ at the center of the sphere, occurs at the time of the big bang after an assumed period of super inflation (proposed by Alan Guth in the early 1980's) where the universe suddenly jumped in size by an enormous factor such as $10^{25}$ times, implying, according to Paul Davies in his book, The Goldilocks Enigma, that "the entire observable universe leapt from about the size of a proton to the size of a grapefruit virtually instantaneously. The actual magnification factor was unimportant so long as it was very big" [1]. He goes on to say, that after this initial instant of time and inflation of size, "inflation shuddered to a halt and normal expansion resumed" [1]. Our beginning model assumes that this normal expansion of the universe (outward along the radii of the sphere) takes place at the speed of light beginning when the universe was about the size of a grapefruit. Thus, a unit of measurement of time, say 1 billion years, is equal to a unit of distance, say 1 billion light years, along both the time axes and the space/distance axes along the surface of the sphere.

In our model, the radius of the sphere is increasing at the speed of light. This is because light travels outward from the beginning of time at the speed of light, so if the age of the universe is about 13.8 billion years old, the radius is at least 13.8 billion light years. We will show later how and why it is much greater than that. It is important to note that the surface of the sphere, which represents the universe, is not expanding at the speed of light. Actually, it is expanding currently by about 7 percent every billion years as indicated by recent observations. We will make calculations on our model later that will produce this same rate of expansion. It is also important to note that the entire universe is on the surface of the sphere in this model. All three space dimensions exist on the surface of the sphere. The $\mathrm{x}$ and $\mathrm{y}$ dimensions extend laterally, and the $\mathrm{z}$ dimension extends perpendicularly to these through the thickness of the sphere, and this will be discussed in more detail later. However, it should be emphasized at this point, that in this model, only time exists inside and outside of the sphere. Past time is inside the sphere, and future time is outside the sphere.

It is important to realize that the orientation of the 3 space directions on the surface of the sphere will depend on the orientation of the observer and local conditions, such as whether the observer, for example, is standing on the earth or floating in space. Thus, all 3 space directions are treated equally, although the thickness direction appears to be much smaller than the other 2 directions. It also appears in the model that we are overlapping the time and space dimensions in the thickness region on the surface of the sphere. This is true, but what does it really mean? Here is where the value of the model becomes evident. Everything inside the sphere is time, past time or history, to be exact. Everything outside of the sphere is also time, which is the future. When someone asks, "What is outside of our universe; i.e., what is outside of the sphere?", the model shows that the simple correct answer is, "It is our future, just as everything inside the sphere is our past." In other words, nothing real exists outside of the sphere, since the outside of the sphere is where the universe (the surface of the sphere) will be in the future. The inside of the universe, so to speak, is where the universe was in the past. The inside is nothing but the past. Everything on the surface of the sphere, including the thickness, is the present time. What this basically says is that space exists in time, and time exists in space. If one asks, "How thick is the thickness of the sphere?”, one is also asking, "How long does present time last?” This is a question for the philosophers, but in our everyday lives, the present time does seem to last for some, although small, period of time before it becomes the past. We will return to this subject later, including a discussion of how thick the thickness is when measurements of height, width or depth are to be made.

We can now place some objects on the model. To keep the size of the model on a single sheet of paper, we can place galaxies spaced one billion light years apart on the surface of the sphere. We can construct the model to show our universe at the present time, which is now widely accepted as approximately 13.8 billion years. For simplicity, we will use 14 billion years as the age of the universe and show the spherical model in cross-section. When time, $t$, is equal to 14 billion years, the circumference of the sphere can be calculated to be approximately 
88 billion light years, $(C=2 \pi r$, or $C=2 \pi(14)$, or $C=87.96)$. Thus there will be 88 galaxies spaced 1 billion light years apart along the circumference of the sphere, which represents the universe. This is not the actual size of our universe at the present time, as we will explain later. We do know that the universe is at least this size, though, since light has been traveling in all directions since shortly after the big bang, at the speed of light, for 13.8 billion years over a distance of at least 13.8 billion light years. The size of the model is now based on the universe expanding at a constant rate (which we will adjust later to show the actual expansion rates), but we will continue to use this simplified version to show how the model works.

The model, as developed so far, is represented in Figure 1. The entire universe is shown on the surface of the sphere. There are 88 galaxies spaced 1 billion light years apart along the surface of the sphere. We will locate our galaxy at the north pole of the sphere. As time proceeds forward, the radius of the sphere increases.

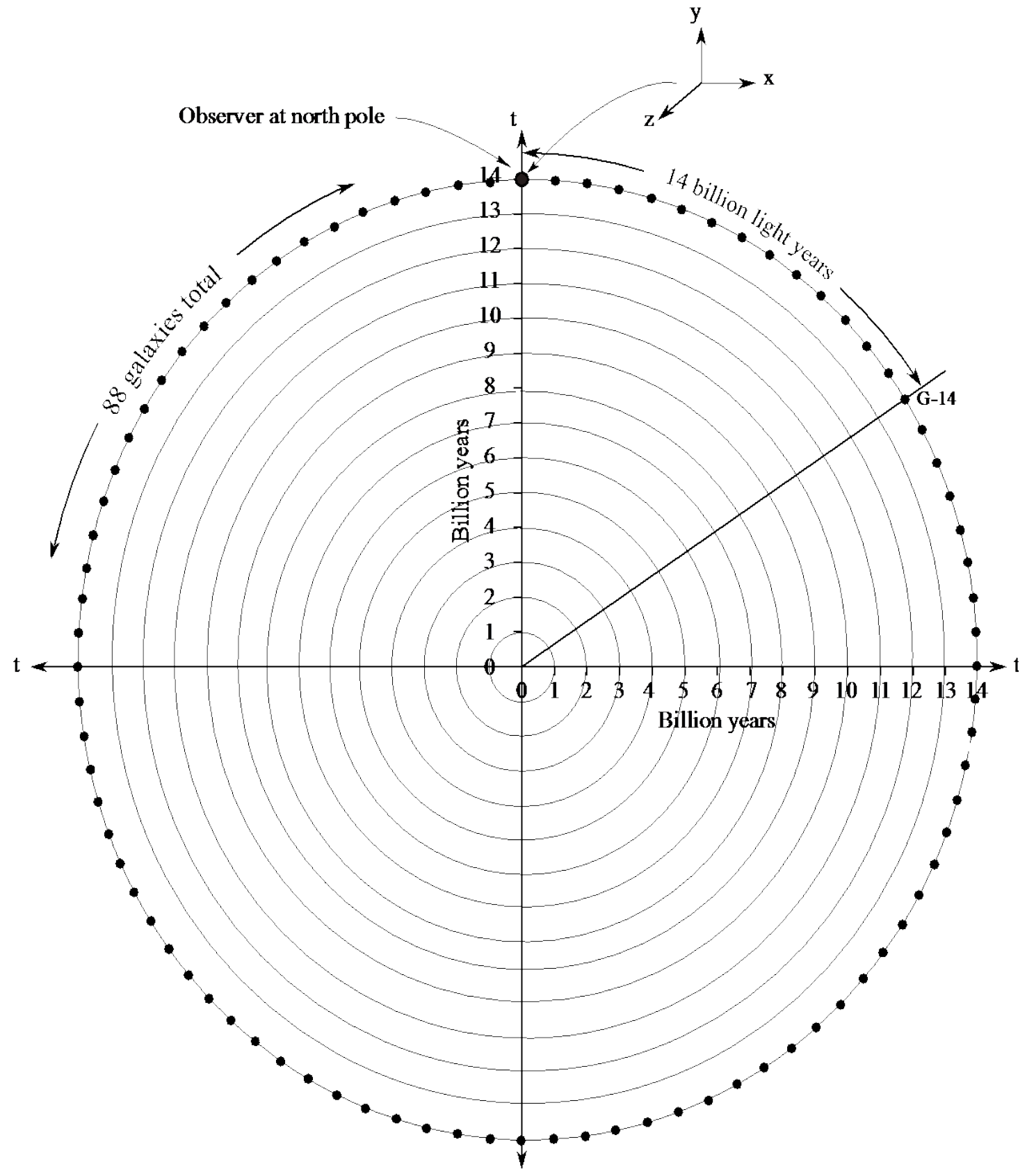

Figure 1. The model. 


\section{The Expanding Universe}

Now that we have established the size of the model representing the universe, we can answer the question of how the model shows how the universe is expanding. The model shows the expansion every billion years by the ever increasing circles in the model that represent the universe as it expands every billion years. For example, if we use the circumference (in cross-section) of our model universe at a time of 14 billion years and compare it to the expanded circumference at a time of say, 15 billion years, the circumference of the sphere (our universe) will have expanded by approximately 7.1 percent. This number is obtained as follows: at $\mathrm{t}=14$ billion years, $C=2 \pi r$, or $C=2 \pi(14)$, or $C=87.9$; at $\mathrm{t}=15$ billion years, $C=2 \pi(15)$ or $C=94.2$; therefore, the percent of expansion of the universe along the surface of the sphere is (94.2 - 87.9) divided by 87.9, which is equal to 0.071 or 7.1 percent. It is interesting to note that Stephen Hawking in his book, A Brief History of Time, states that the universe is expanding by 5 to 10 percent every 1 billion years, based on measurements of the velocities at which other galaxies are moving away from us, using the Doppler effect [2]. What is even more interesting is that the Hubble constant, which is the current value used to determine the universes' expansion, agrees almost exactly with the above number. In a paper titled "Is the universe expanding faster than the speed of light?", Dave Rothstein from the Astronomy Department at Cornell University states that the Hubble constant "is approximately equal to 71, measured in the technically useful but conceptually confusing units of 'kilometers per second per megaparsec'. In more sensible units, the Hubble constant is approximately equal to $0.007 \%$ per million years-what it means is that every million years, all the distances in the universe stretch by $0.007 \%$ " [3]. This value is the same as $7 \%$ every billion years. His paper was written in September 2003. The present value for the Hubble constant, as determined by the Planck space mission and reported in March 2013, is now just under 68.

It should be noted that if, for example, our universe were twice as big and its circumference were 176 billion light years instead of 88 billion light years, the circumference would have to expand by approximately 12.3 billion light years over 1 billion years (instead of 6.3 billion light years) to equal 0.070 or 7 percent. This shows that the model can be easily adjusted to reflect actual experimental data.

Our model clearly shows how the size of the universe is expanding outwardly at the speed of light into the future (i.e., 14 billion years of time is equivalent to 14 billion light years of distance), while the galaxies along the surface of the sphere (circumference in cross-section) are moving away from each other by approximately 7 percent over 1 billion years as we observe today. The model reflects reality in that, as we look out into space, we are looking back in time 14 billion years to the time of the big bang. As we do this, we are also looking out into space a distance of 14 billion light years. It is logical then, that the size of the universe (determined by the radius) can be expanding at approximately the speed of light while the galaxies are moving apart from each other by only 7 percent over 1 billion years as we observe today. Since this value corresponds with the actual value of approximately 7 per cent over 1 billion years, the model appears to be an accurate model.

The model shows, that if we choose a galaxy, galaxy G-7, that is now 7 billion light years away from our galaxy, located in our model on the surface of the sphere at a time of 14 billion years, it would have been 3.5 billion light years away from our galaxy 7 billion years ago at time $t$ equals 7 billion years. The light emitted from that galaxy 7 billion years ago will travel toward our galaxy at the speed of light through an expanding universe presented by the expanding spheres in the model. We can calculate the distance light will have traveled every 1 billion years on the expanding spheres and show it on the model.

We first have to show how much the universe has expanded every billion years in the model. At time, $t=7$ billion years, the circumference of the universe would be $2 \pi r$, or $2 \pi(7)$, which equals 43.96 billion light years. At time, $\mathrm{t}=8$ billion years, the circumference would be $2 \pi(8)$ which equals 50.24 billion light years. The expansion is thus, 50.24 minus 43.96, or 6.28 billion light years. The distance increase in expansion per billion light years at time, $\mathrm{t}=8$ billion years, is 6.28 billion light years divided by 43.96 billion light years, or 0.1429 billion light years. We can make similar calculations for each year as the universe expands from 8 billion years old to 14 billion years old. While we are doing this, we can also make the similar calculations during the first 7 billion years of the universe's expansion. We are assuming that the expansion occurs at a constant rate during this time period; i.e., the size of the universe, as determined by the radius, increases by 1 billion light years every 1 billion years. We will show later how the model can depict the expansion at the rates determined by current experimental data.

Now that we have calculated how much the universe has expanded from a time of 7 billion years to 8 billion years, we can determine how far the emitted light has traveled during this 1 billion year time period. 


\section{Light Traveling through Expanding Space}

To do this, we need to know how light travels through expanding space. For example, if 2 galaxies are 1 billion light years apart, and after 1 billion years, they are now 1.1 billion years apart due to the expansion of space, how far did light travel when it was emitted from one of the galaxies 1 billion years ago? We can show by analogy presented in Appendix A, that light would have traveled the 1 billion light years (the speed of light is 1 billion light years per billion years) plus 1/2 the expanded distance. It thus would have traveled 1.05 billion light years and still be 0.05 billion light years away from the other galaxy.

We can now return to our previous example and determine how far the emitted light has traveled during this 1 billion year time period from time, $t=7$ to time, $t=8$. It is simply 1 billion light years plus $1 / 2$ the expanded distance, or 1 billion light years plus $1 / 2$ of 0.1429 light years or 1.0714 billion light years. We can again make similar calculations for the remainder of the time from 8 billion years to 14 billion years, and also, all the way back to the beginning of the universe. We will begin the calculations at a time of 380 thousand years after the big bang, which is now considered to be the time at which light was first emitted from the early universe. To be consistent, we started our calculations for the expansion of the universe at the same time, 0.00038 billion years, which is practically zero on the scale of our model. If the universe expanded at a constant rate of 1 billion light years per 1 billion years during its first billion years, the expansion per billion years is approximately 1 billion light years. The distance light traveled during this time period is the distance it traveled at the speed of light from the time light was first emitted to 1 billion years, or approximately 1 billion light years, plus $1 / 2$ the expanded distance ( $1 / 2$ of 1 billion light years), or a total of 1.5 billion light years. These calculations are shown in Table 1.

Using Table 1, we can now show how the light emitted from galaxy, G-7, at a time of 7 billion years travels toward our galaxy. We have plotted the light beginning at time, $t=7$, and calculated what the remaining distance

Table 1. Expansion at a constant rate.

\begin{tabular}{|c|c|c|c|c|c|c|}
\hline \multirow{2}{*}{$\begin{array}{c}\text { Time, t } \\
\text { (BY) }\end{array}$} & \multirow{2}{*}{$\begin{array}{c}\text { Radius, r } \\
\text { (BLY) }\end{array}$} & \multicolumn{2}{|c|}{$\begin{array}{l}\text { Circumference, C } \\
\text { (BLY) \& diff. }\end{array}$} & \multirow{2}{*}{$\begin{array}{c}\begin{array}{c}\text { Expansion per BLY, } \\
(\mathrm{BLY})^{*}\end{array} \\
0.0769\end{array}$} & \multirow{2}{*}{$\begin{array}{c}\begin{array}{c}\text { Distance light } \\
\text { traveled (BLY) }\end{array} \\
1.0385\end{array}$} & \multirow{2}{*}{$\begin{array}{c}\text { \% Expansion } \\
\\
7.7\end{array}$} \\
\hline & & 87.90 & 6.28 & & & \\
\hline 13 & 13 & 81.64 & 6.28 & 0.0833 & 1.0417 & 8.3 \\
\hline 12 & 12 & 75.36 & 6.28 & 0.0909 & 1.0455 & 9.1 \\
\hline 11 & 11 & 69.08 & 6.28 & 0.1000 & 1.0500 & 10.0 \\
\hline 10 & 10 & 62.80 & 6.28 & 0.1111 & 1.0555 & 11.1 \\
\hline 9 & 9 & 56.52 & 6.28 & 0.1250 & 1.0625 & 12.5 \\
\hline 8 & 8 & 50.24 & 6.28 & 0.1429 & 1.0714 & 14.3 \\
\hline 7 & 7 & 43.96 & 6.28 & 0.1667 & 1.0833 & 16.7 \\
\hline 6 & 6 & 37.68 & 6.28 & 0.2000 & 1.1000 & 20.0 \\
\hline 5 & 5 & 31.40 & 6.28 & 0.2500 & 1.1250 & 25.0 \\
\hline 4 & 4 & 25.12 & 6.28 & 0.3333 & 1.1667 & 33.3 \\
\hline 3 & 3 & 18.84 & 6.28 & 0.5000 & 1.2500 & 50.0 \\
\hline 2 & 2 & 12.56 & 6.28 & 1.0000 & 1.5000 & 100 \\
\hline 1 & 1 & 6.28 & 6.28 & 1.0000 & 1.5000 & 100 \\
\hline $0+$ & 0.00038 & 0.002 & N/A & N/A & N/A & N/A \\
\hline & & & & & 16.0901 total & \\
\hline
\end{tabular}

BY = billion years. BLY = billion light years. ${ }^{*}$ Increase in circumference divided by the previous circumference. ${ }^{* *}$ Increase in circumference divided by the previous circumference in percent. 
is to our galaxy as it travels through expanding space. For example, as the emitted light travels from time, $\mathrm{t}=7$ to $t=8$, we calculate the total distance the universe expanded between our galaxy and the place at which the light was emitted during this time, which is 3.5 billion light years, plus ( 0.1429 times 3.5 billion light years), and then subtract the distance light traveled, 1.0714 billion light years, during this time period. The result is 2.9288 billion light years remaining to our galaxy. Similar calculations can be performed as the light continues to travel toward our galaxy each billion years. These calculations are shown in Table 2. The table shows that the emitted light will have reached our galaxy after traveling through expanding space for 4.52 billion years, at a time of 11.52 billion years. This is also shown in Figure 2.

\section{Our Visible Universe}

Now that we know how the stage 1 model represents the universe and know how calculations are made with it, we can show how the model describes our visible universe. Remember, our entire universe, as it exists now, is shown on the surface (circumference in cross-section) of the sphere at a time of $t=14$ billion years. Our visible universe will be shown on the model as a shape determined by the light beams now reaching our galaxy from all the galaxies in the universe from the time they first emitted light. Just as in the previous example where we plotted the light emitted 7 billion years ago from galaxy, G-7, to where it reached our galaxy a little over 2 billion years ago, we can begin plotting the light which we are now receiving at our galaxy at a time of 14 billion years, and trace it backwards in space and time on the model. We can use the calculations presented in Table 1 to plot the light back to a time of 380 thousand years after the big bang. This is shown in Figure 3 . The shape of the visible universe appears as a tear drop shaped figure in cross section, with a slightly indented bottom, in 3 dimensions (2 space and 1 time).

When we look far out into space with our telescopes, we are seeing galaxies as they existed billions of years ago. We can, for example, select galaxy G-28, which is now 28 billion light years away from our galaxy, and determine by our model that it is now in our visible universe, and we are just now seeing it as it existed when our universe was about 2 billion years old. We can also determine that our galaxy and galaxy G-28 were about 4 billion light years apart when the light which we are seeing was emitted from galaxy G-28. (This is the distance between the 2 galaxies measured along the circumference at a radius of 2 billion light years). The visible universe in the model is a tear drop shaped globe that extends from our galaxy out in all directions along the surface of the globe for a distance of 16.09 billion light years.

Now that we have created our basic stage 1 model, we can make some adjustments to it. We mentioned earlier that we developed the model based on our universe expanding at a constant rate. Data obtained recently by telescope shows that the expansion of the universe was actually decelerating for its first 8 billion years, and then the expansion began to accelerate. Zosia Rostomian, Lawrence Berkeley National Laboratory, and Nick Ross, BOSS Lyman-alpha team, Berkeley Lab, have developed a graph based on data from a spectroscopic survey

\section{Table 2. Light emitted from G-7 at time, $\mathrm{t}=7$ billion years.}

\begin{tabular}{ccccccc}
\hline $\begin{array}{c}\text { Time, } \mathrm{t} \\
(\mathrm{BY})\end{array}$ & $\begin{array}{c}\text { Radius, } \mathrm{r} \\
(\mathrm{BLY})\end{array}$ & \multicolumn{2}{c}{$\begin{array}{c}\text { Circumference, } \mathrm{C} \\
\text { \&diff. (BLY) }\end{array}$} & $\begin{array}{c}\text { Expansion per } \\
\text { BLY, (BLY) }\end{array}$ & $\begin{array}{c}\text { Distance light } \\
\text { traveled (BLY) }\end{array}$ & $\begin{array}{c}\text { Distance } \\
\text { remaining (BLY) }\end{array}$ \\
\hline 14 & 14 & 87.90 & 6.28 & 0.0769 & 1.0385 & \\
13 & 13 & 81.64 & 6.28 & 0.0833 & 1.0417 & -0.4811 \\
12 & 12 & 75.36 & 6.28 & 0.0909 & 1.0455 & 0.5174 \\
11 & 11 & 69.08 & 6.28 & 0.1000 & 1.0500 & 1.4249 \\
10 & 10 & 62.80 & 6.28 & 0.1111 & 1.0555 & 2.2324 \\
9 & 9 & 56.52 & 6.28 & 0.1250 & 1.0625 & 2.9288 \\
7 & 8 & 50.24 & 6.28 & 0.1429 & 1.0714 & 3.5 \\
\hline
\end{tabular}

$\mathrm{BY}=$ billion years. BLY = billion light years. ${ }^{*}$ Increase in circumference divided by the previous circumference. 


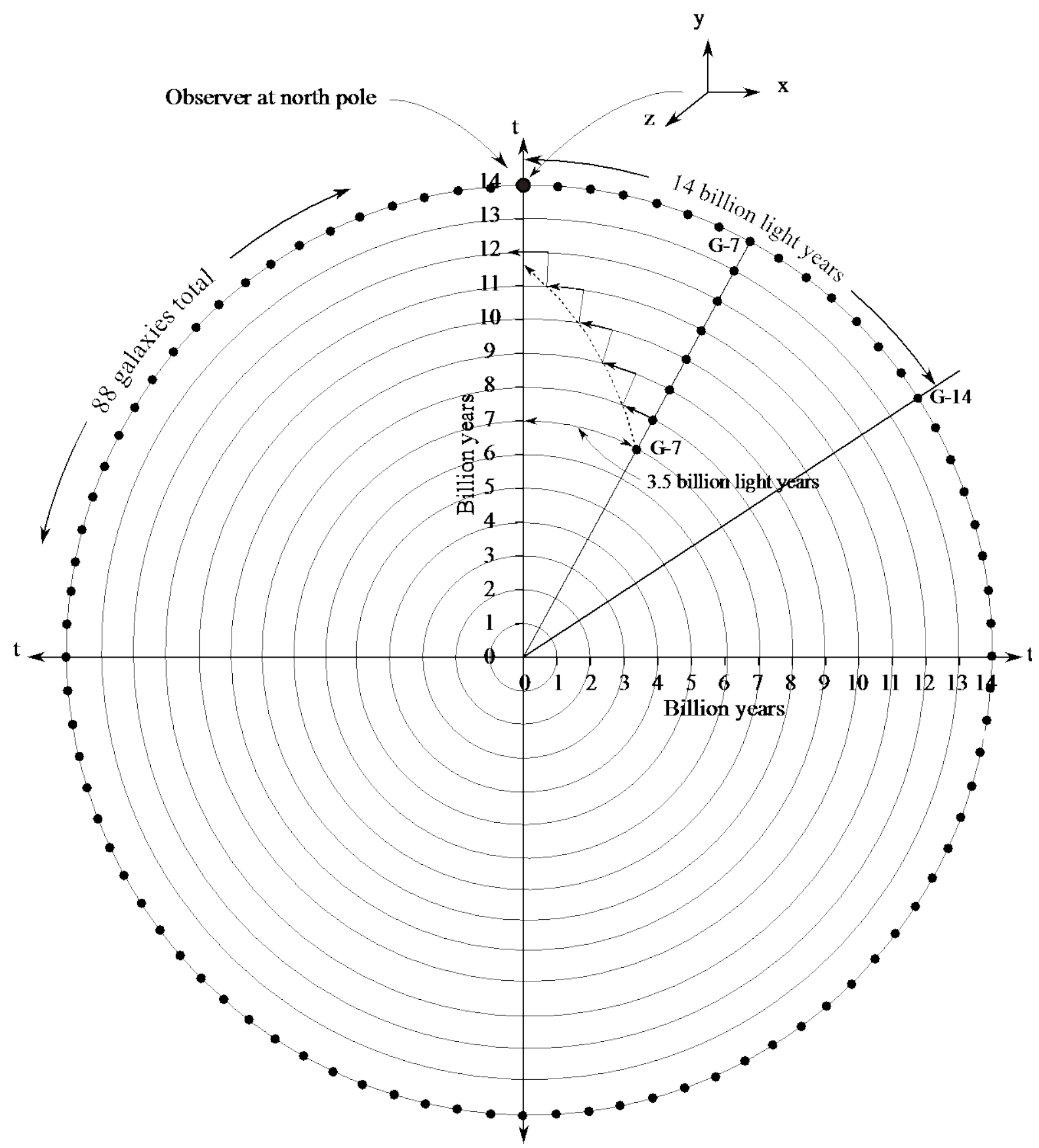

Figure 2. Light emitted from galaxy G-7.

(Boss Sloan Digital Sky Survey-III, November, 2012) that depicts this changing expansion [4]. This graph is shown in Figure 4.

We can change our model to mirror the data in this graph by increasing or decreasing the size of our model (circumference in cross-section) each billion years to match the deceleration and acceleration shown in the graph. For example, to show the rapid decrease in expansion during the first billion years, the size of the universe, as determined by the radius of the model, had to be expanding by billions of light years during the first billion years. Then, as the deceleration slowed down and leveled out at about a time of 8 billion years, the radius was expanding at a rate considerably slower than its current rate of about $6.9 \%$ every billion years. The expansion then began to slowly accelerate. The radius of the expanded model has to be large enough to mirror this expansion. 


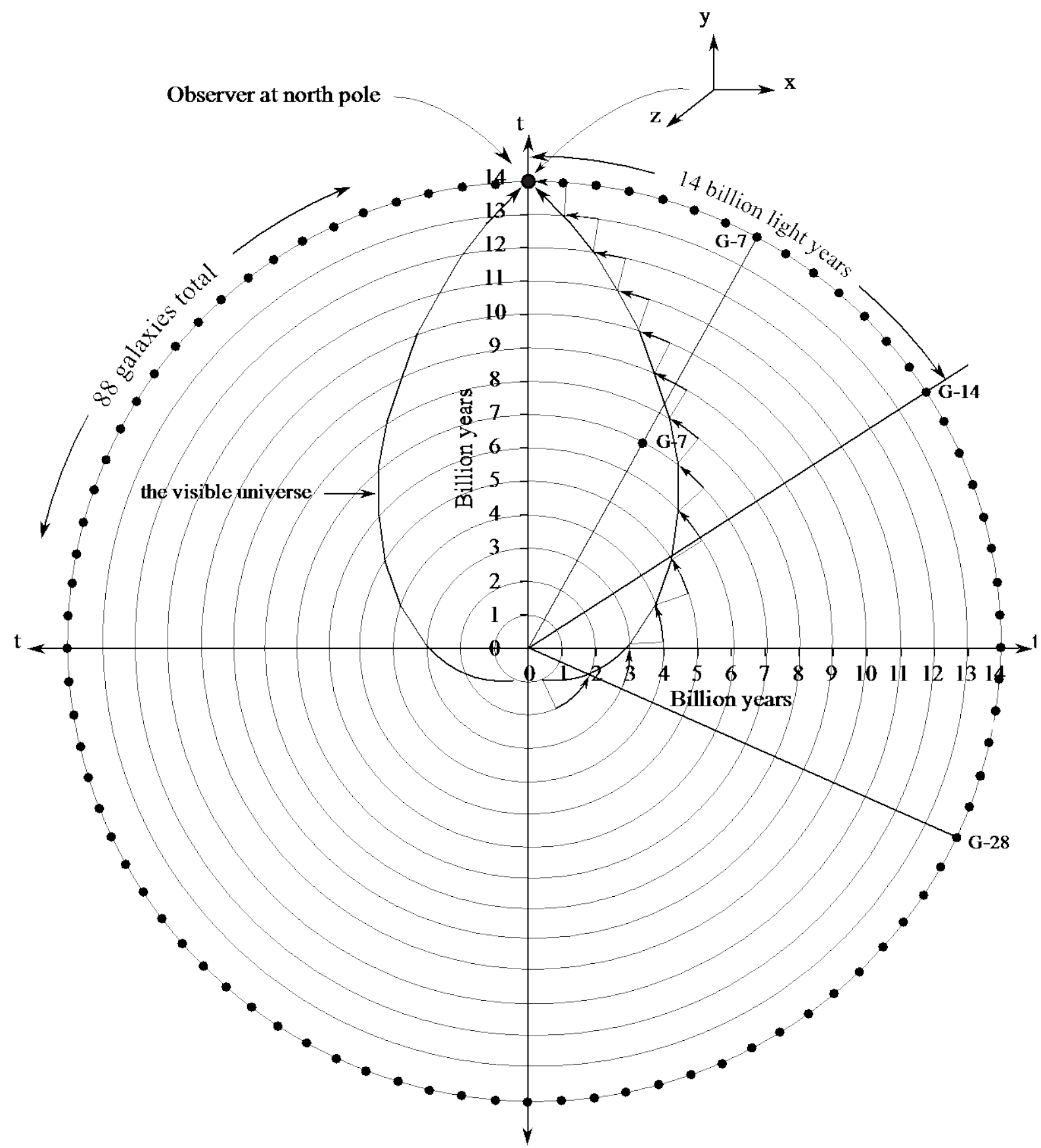

Figure 3. The visible universe.

It also has to reflect current consensus about the size of our visible universe. The most important for our model is the general agreement in the literature that the galaxies that we can observe when the universe was less than a billion years old, and their light has taken over 17 billion years to reach us, are now about 46 billion light years away from us. This is shown as galaxy, G-46 on the model. To reflect this result, the radius of the model has to be about 40 billion light years. This is all shown in Table 3, in column 2 (change in radius), and in column 6 (\% Expansion). It is also shown in Figure 5. It should be noted that at a time of 14 billion years (now), the model shows that the universe is expanding at a rate of $6.9 \%$, which relates very closely to the current value of the Hubble constant.

The model in Figure 5 shows both the new time and corresponding radius measurement on the vertical axes. 


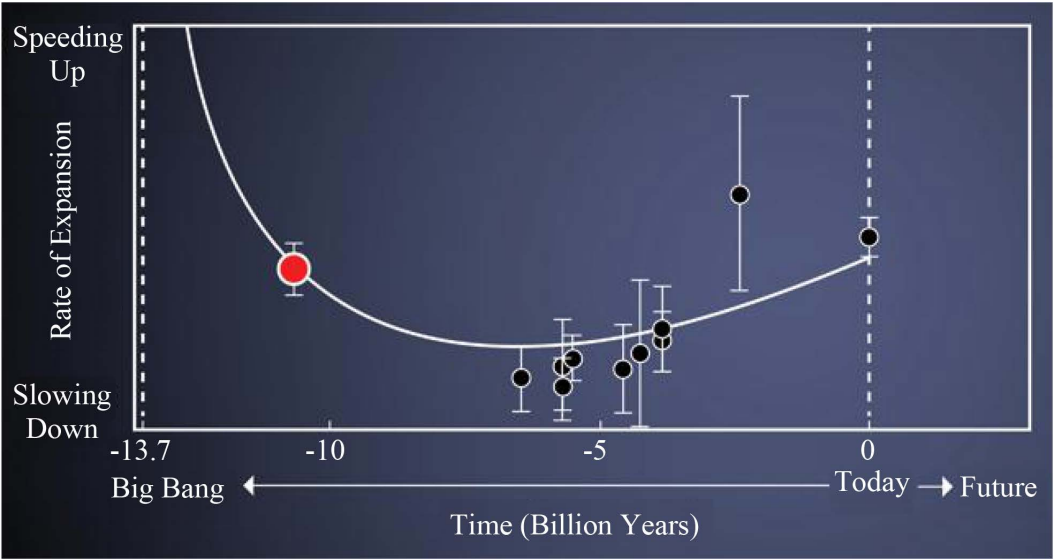

Figure 4. Expansion of the universe (Credit: Zosia Rostomian, LBNL; Nic ross, Boss lyman-alpha team, LBNL).

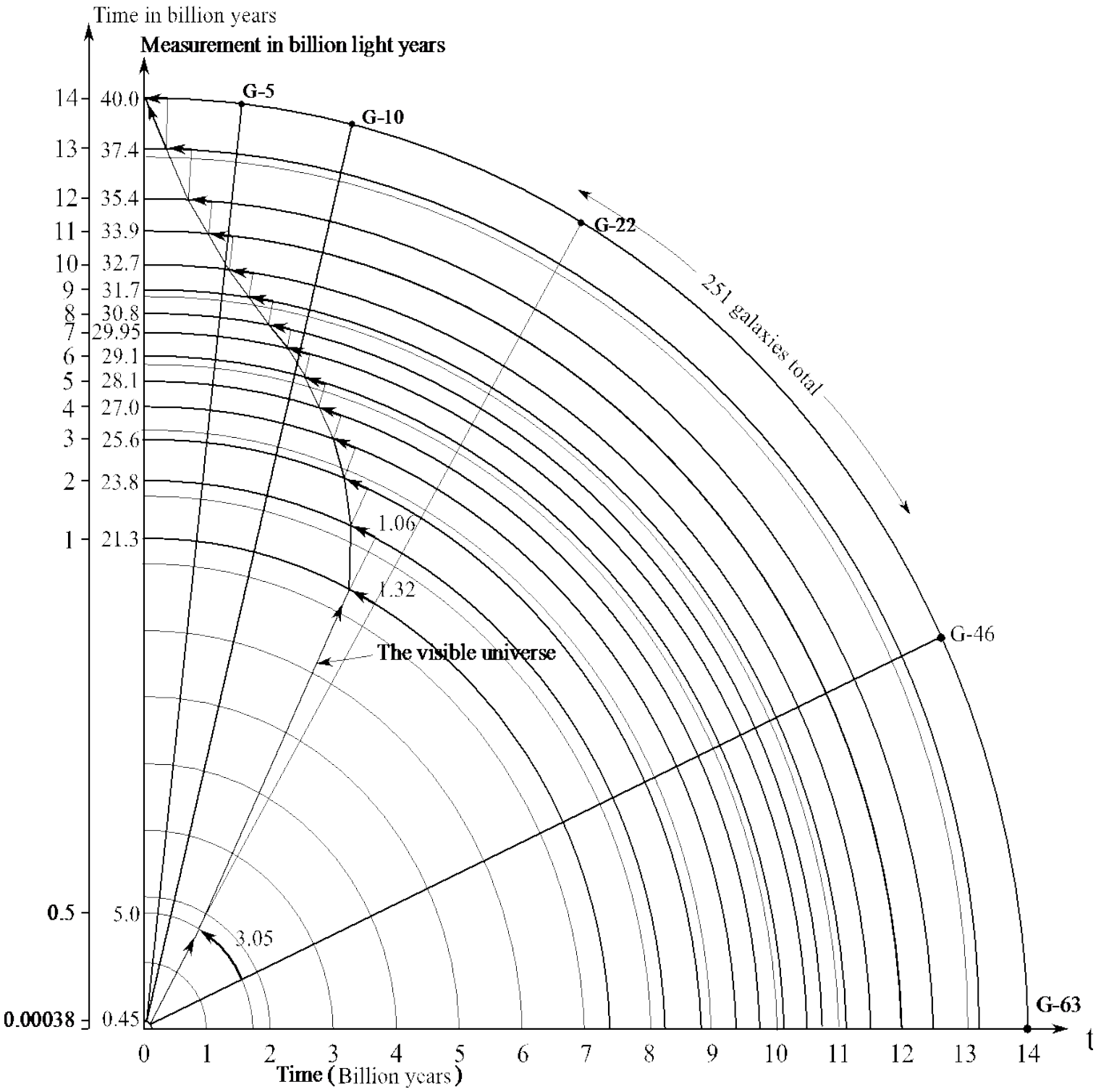

Figure 5. Expansion of the universe at observed rate. 
Table 3. Expansion at observed rate.

\begin{tabular}{|c|c|c|c|c|c|c|}
\hline \multirow{2}{*}{$\begin{array}{c}\text { Time, t } \\
(\mathrm{BY})\end{array}$} & \multirow{2}{*}{$\begin{array}{c}\begin{array}{c}\text { Radius, r } \\
\text { (BLY) }\end{array} \\
40.0\end{array}$} & \multicolumn{2}{|c|}{$\begin{array}{l}\text { Circumference, C } \\
\text { (BLY) \& diff. }\end{array}$} & \multirow{2}{*}{$\begin{array}{c}\text { Expansion per } \\
\text { BLY, (BLY) } \\
0.0694\end{array}$} & \multirow{2}{*}{$\begin{array}{c}\begin{array}{c}\text { Distance light } \\
\text { traveled (BLY) }\end{array} \\
1.0347\end{array}$} & \multirow{2}{*}{$\begin{array}{c}\begin{array}{c}\% \\
\text { Expansion }^{* *}\end{array} \\
6.9\end{array}$} \\
\hline & & 251.2 & 16.3 & & & \\
\hline 13 & 37.4 & 234.9 & 12.6 & 0.0567 & 1.0284 & 5.7 \\
\hline 12 & 35.4 & 222.3 & 9.4 & 0.0442 & 1.0221 & 4.4 \\
\hline 11 & 33.9 & 212.9 & 7.5 & 0.0365 & 1.0183 & 3.7 \\
\hline 10 & 32.7 & 205.4 & 6.3 & 0.0316 & 1.0158 & 3.2 \\
\hline 9 & 31.7 & 199.1 & 5.7 & 0.0295 & 1.0148 & 3.0 \\
\hline 8 & 30.8 & 193.4 & 5.3 & 0.0282 & 1.0141 & 2.8 \\
\hline 7 & 29.95 & 188.1 & 5.4 & 0.0296 & 1.0148 & 3.0 \\
\hline 6 & 29.1 & 182.7 & 6.2 & 0.0351 & 1.0176 & 3.6 \\
\hline 5 & 28.1 & 176.5 & 6.9 & 0.0407 & 1.0204 & 4.1 \\
\hline 4 & 27.0 & 169.6 & 8.8 & 0.0547 & 1.0274 & 5.5 \\
\hline 3 & 25.6 & 160.8 & 11.3 & 0.0756 & 1.0378 & 7.6 \\
\hline 2 & 23.8 & 149.5 & 15.7 & 0.1173 & 1.0587 & 11.7 \\
\hline 1 & 21.3 & 133.8 & 102.4 & 3.2611 & 1.3153 & 326 \\
\hline 0.5 & 5.0 & 31.4 & 28.6 & 10.20 & 3.0500 & 1020 \\
\hline 0.00038 & 0.45 & 2.8 & 2.8 & N/A & N/A & N/A \\
\hline \multirow[t]{2}{*}{0} & 0 & 0 & 0 & N/A & N/A & N/A \\
\hline & & & & & 17.6902 & \\
\hline
\end{tabular}

BY = billion years. BLY = billion light year. ${ }^{*}$ Increase in circumference divided by previous circumference. ${ }^{* *}$ Increase in circumference divided by the previous circumference in percent per BLY.

Note that at a time of 380,000 years when light was first emitted throughout our universe, its radius was 450 million light years. The galaxies that were just being formed at that time are now about 46 billion light years away from our galaxy. They are represented by galaxy G-46 on the model. Galaxies that are more distant than that, such as galaxy G-63, are not in our visible universe. We have also shown galaxy G-5 that was in our visible universe at a time of 10 billion years, galaxy G-10 at a time of 6.5 billion years, and galaxy G-22 at a time of 0.5 billion years. These will be used later when timelines are needed.

\section{The Fourth Space Dimension}

The next adjustment we can make on the model presents us with a paradigm shift in how we view our universe. Earlier, when we first developed our model, we described how all 3 space dimensions exist on the surface of the sphere and how the height dimension (thickness of the sphere) also represents the present time. To develop the true height dimension at any point on the sphere, we can rotate the sphere at that point.

If we look at Figure 1, which we will now call stage 1 of the model, and locate ourselves at the north pole of the sphere at $\mathrm{t}=14$ billion years, we can visualize the universe as the surface of the sphere in 2 lateral dimensions. To obtain the third dimension; i.e., the height dimension, we can rotate the sphere 90 degrees about the north pole, and now the 2 lateral dimensions include the height dimension. As we rotate the sphere, it is important that we maintain our true distances along the surface of the sphere, which means, for example, that the distance of 17.69 billion light years from our galaxy to the most distant galaxy in our visible universe (and actually, back to the time light was first emitted in our universe about 380,000 years after the big bang) will become the new radius of our rotated sphere. We are changing the orientation of the sphere so that the original "up" direction is now pointing in the left horizontal direction in Figure 6. The important point is that this 4-space dimension universe is now experiencing the two different orientations at the same time. As 3 dimensional beings, we cannot do the same. We should also note that as the orientation of "up" changed, the center of the universe at $\mathrm{t}=$ 


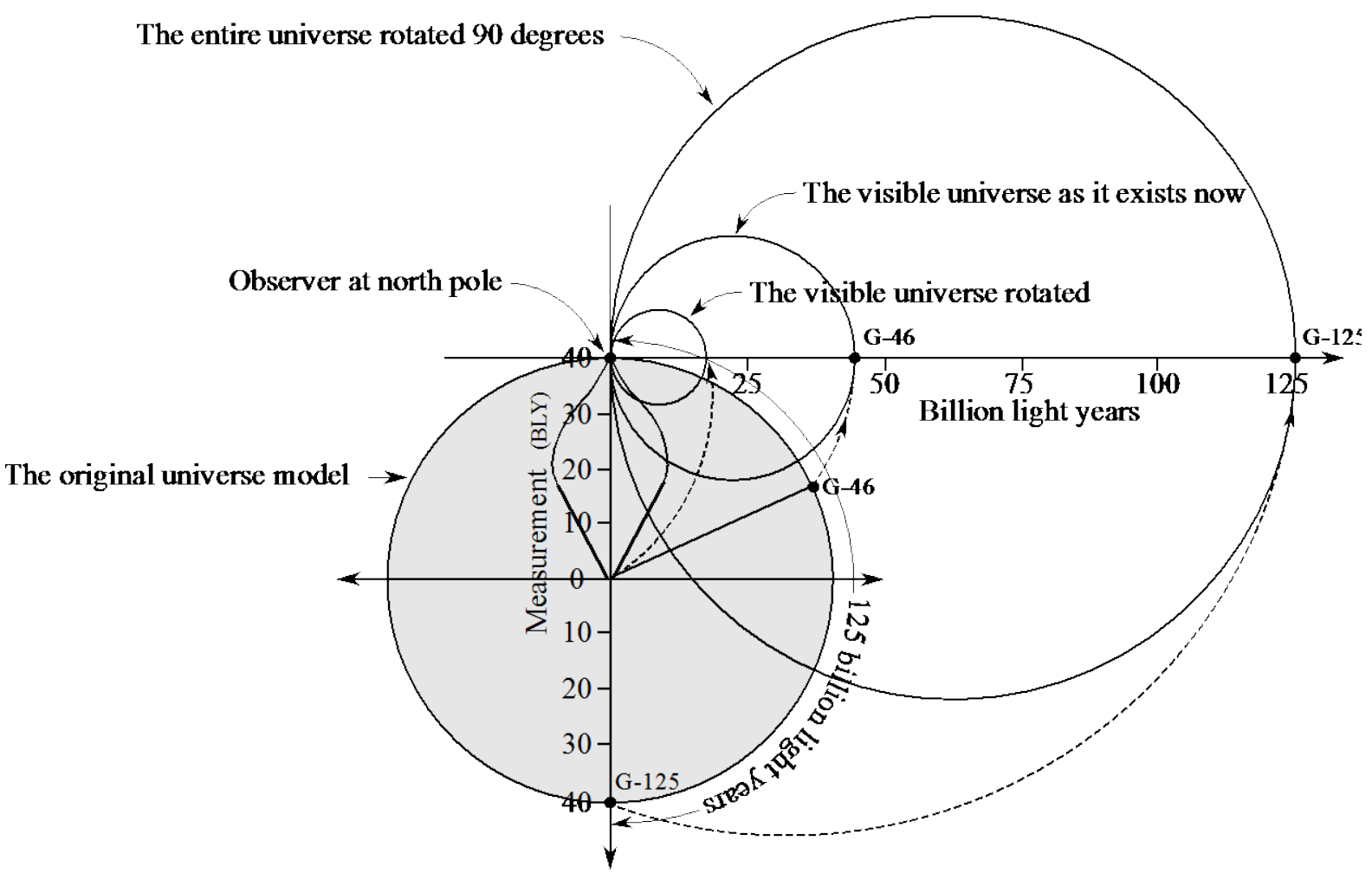

Figure 6. The universe rotated 90 degrees.

0; i.e., the big bang, changed so that it is now also located in a horizontal direction to the right from the north pole as in Figure 6.

In Figure 6, we are not rotating the entire original universe model. We are rotating the spheres, which represent distances and space, into the radii which represent time, at the location of the observer at a single time of 13.8 billion years. We are rotating the surface of the sphere representing the entire universe which will have a radius of 125 billion light years, and the surface of the visible universe as it exists now, which will have a radius of 46 billion light years. Each will have the same timeline, as shown on the vertical axis in Figure 5, on its radius which we will show after its complete rotation in Figure 7. The rotation of the original visible universe is more complicated because it includes both distance and time, not just distance. The original visible universe represents the distances light traveled during 14 time periods from the observer back to 380,000 years. It is a representation of the distances light traveled over time which is speed, or velocity. The actual distances light traveled are the arrows on the 14 different circumferences in Figure 5 that add up to a total distance of 17.69 billion light years. This is what is being rotated and shown in Figure 6. It will have its own timeline which stretches back to 380,000 years.

If we now change the "up" direction to all possible directions by rotating the spheres about the north pole in all possible directions, and the universe experiences all these different orientations at the same time, the model will now represent the entire universe with its center at the north pole. In addition, the surface of the initial universe will now have completely populated the volume of the large sphere, shown as the entire universe, as shown in cross-section in Figure 7. By changing the orientation of the surface of the sphere to all possible orientations, we have picked up all the galaxies that exist throughout the universe. By rotating the surface of the sphere (space) in all possible directions (time), we have merged space with time and developed 4-dimensional space-time which we experience in our real world today.

By using orientation as a $4^{\text {th }}$ space dimension, our model, which we can now call stage 2 , now reflects our 3-space dimension world as we know it. We are 3-dimensional beings who can experience only one orientation (or "up" direction) at a time. We cannot experience all possible orientations at the same moment in time; whereas the universe does "experience" all possible orientations at the same time as shown in Figure 7. We are 3space dimensional beings living in a 4-space dimensional universe. This is analogous to the 2-dimensional 


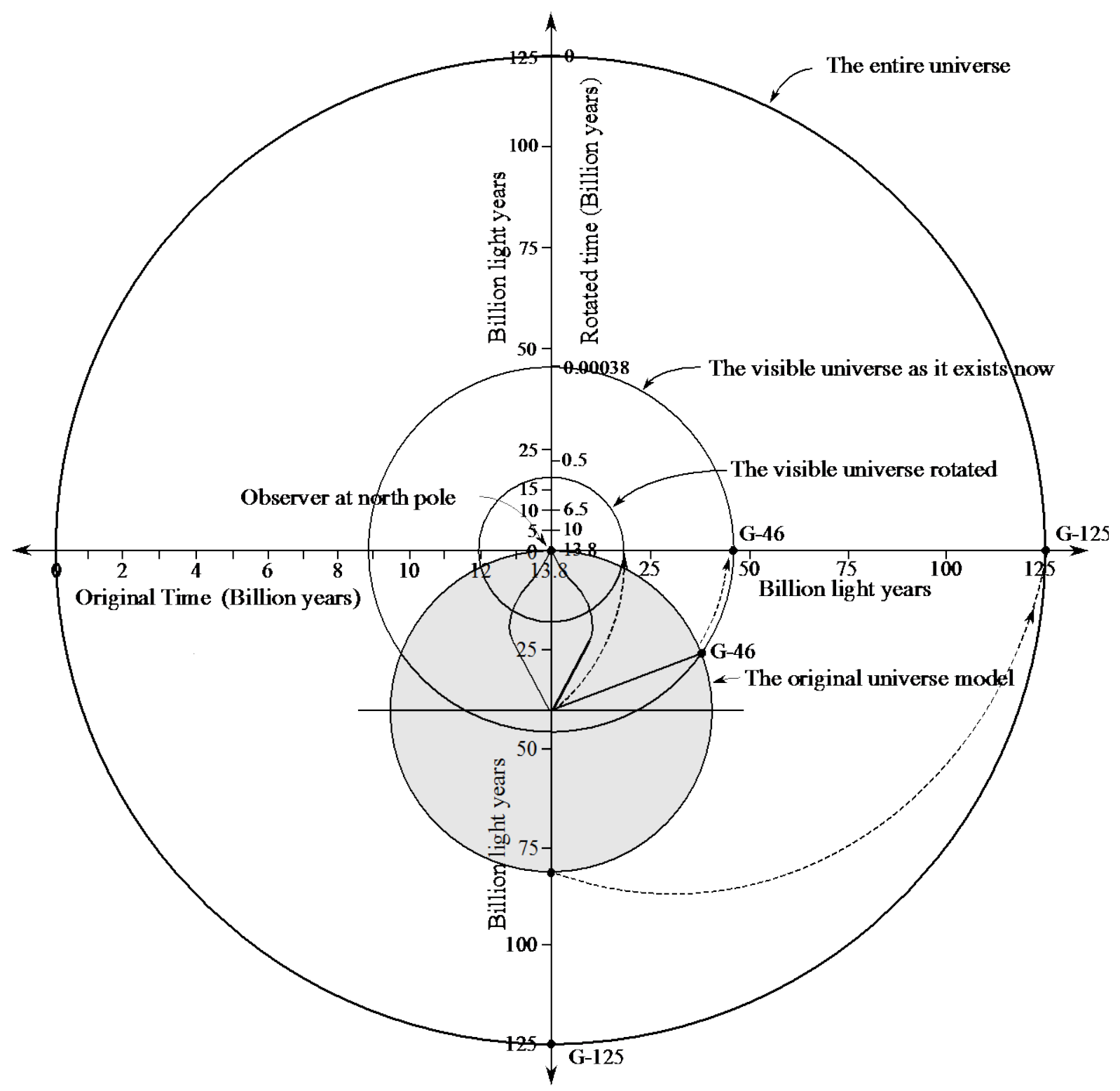

Figure 7. The stage 2 model with 4 space dimensions.

being living on a horizontal plane at one arbitrary height level who cannot experience the third dimension of height since there is no "up" direction in his flat 2-dimensional world. There are only 2 horizontal directions. In other words, in a 2-dimensional world, an intelligent square cannot become, or even conceptualize becoming a cube since the flat square can experience only one height level at a time. Being 2-dimensional, the square cannot experience many height levels at the same time, even though they may exist all around the square, or else the square could then become a 3-dimensional cube and no longer be 2-dimensional.

The model shown in Figure 7 includes 4 space dimensions (width, height, depth, and orientation) and the time dimension. The timeline for the rotated distances of the entire universe, and the rotated distances of the visible universe as it exists now, are shown on the right side of the top of the vertical axis. They are based on the distances to galaxies G-5, G-10, G-22, and G-46 at the times at which they appeared in our visible universe as presented in Figure 5. For example, in Figure 5, the distance to galaxy G-10 along the circumference of the sphere at a time of 14 billion years (now) is 10 billion light years. When we track it back in time to where it intersects the line representing our visible universe, it appears in our visible universe at a time of 6.5 billion years (on the far left vertical axis). Thus, in Figure 7, a time of 6.5 billion years corresponds to a distance from the 
observer (our galaxy) of 10 billion light years. Likewise, the distance of 46 billion light years to galaxy G-46 corresponds to a time of 380,000 years after the big bang, and the farthest distance to galaxy G-125 corresponds to a time of zero at the big bang. These last two distances and times are the numbers that will be used in the calculations to explain dark matter and dark energy. Another version of the model is shown in Figure 8, again in cross-section.

In Figure 8, the volume of the outer sphere represents our entire universe. It has a radius of 125 billion light years. The surface of the outer sphere represents the time of the creation of the universe, the big bang, at a time of 13.8 billion years ago. The volume of the middle sphere (The visible universe as it exists now, in Figure 8) represents all the galaxies in our visible universe as they exist now. It has a radius of 46 billion light years. This shows that the galaxies which were being formed, and we now observe just after the time of recombination at a time of 380,000 years, (and it has taken 17.7 billion years for their light to reach us), are now about 46 billion light years away from us. The volume of the smallest sphere (The visible universe rotated, in Figure 8) represents our visible universe which we can observe in our telescopes. It has a radius of 17.7 billion light years. The surface of this sphere also represents the time of recombination when light was first emitted in the universe,

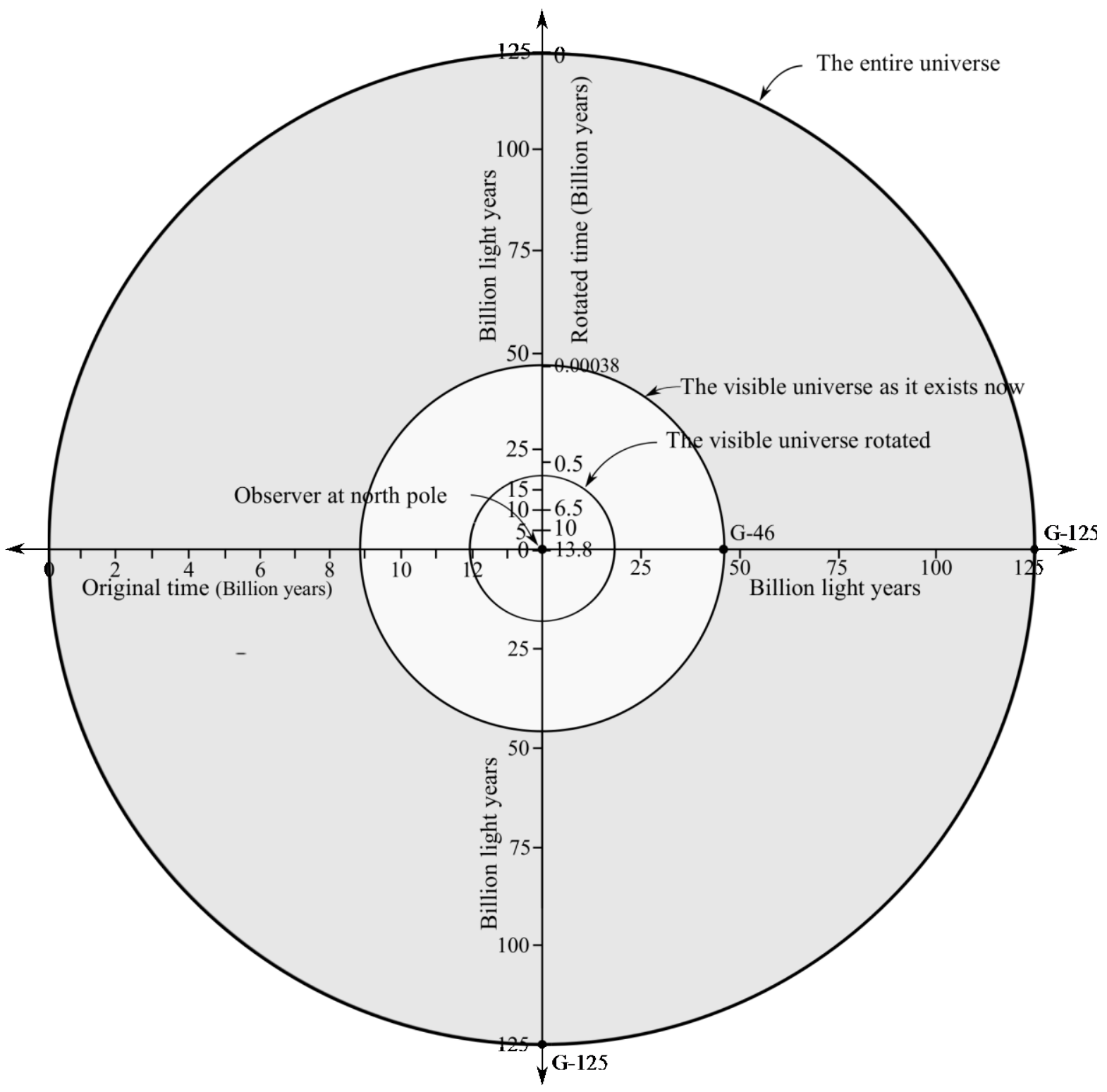

Figure 8 . The simplified version of the stage 2 model. 
at a time of 380,000 years after the big bang.

We can now make some calculations. We can compare the volume of the visible universe as it exists now (not as it existed in the past represented by the smaller visible universe), to the volume of the entire universe as it exists now. This will then give us the percentage of visible matter (baryonic) that we can now detect in our visible universe, compared to the amount of baryonic matter in the entire universe. Using the formula, $V=\frac{4}{3} \pi r^{3}$, the volume of the visible universe as it exists now, with a radius of $r=46$ billion light years, is 407,513 cubic billion light years. The volume of the entire universe, with a radius of $r=125$ billion light years, is 8,177,083 cubic billion light years. The ratio is thus, 20.7. This is almost exactly the same as the ratio of visible matter in the visible universe determined by the Planck space mission, compared to the total of all matter, including dark matter and dark energy, in the universe. Recent NASA Planck space mission data reported in March 2013 show that the early universe was 4.9 percent real (visible) matter in the form of atoms, 26.8 percent unseen dark matter and 68.3 percent unseen dark energy. Thus, the ratio of visible matter to all matter in the universe including dark matter and dark energy is 100 divided by 4.9, or 20.4. The model explains what the dark matter and dark energy is. It is the rest of the normal matter in the entire universe that is not visible to us because it is not in our visible universe.

We can now discuss dark matter and dark energy, but before we do, we should look again at the model in Figure 8. The dark area between the visible universe as it exists now, and the entire universe, contains the real matter (baryonic) that exists in the dark area that produces the gravity in our visible universe that we refer to as dark matter and dark energy. Dark matter and dark energy are in our visible universe simply as gravity, not as matter. This will be discussed in detail later. We know they are there because of their gravitational effects upon the stars in our galaxies and the accelerated expansion of the universe. They make up 20.7 times the amount of visible matter in our visible universe. The model also shows how time (on the top half of the vertical axis) extends from zero at the time of the big bang at a distance of 125 billion light years away from our galaxy, to a time of 0.00038 billion years at the beginning of our visible universe as it exists now at a distance of 46 billion light years away from our galaxy, to a time of 13.8 billion years after the big bang at our galaxy.

This stage 2 model reflects the reality of our world in several striking ways. Have you wondered why, for example, when the Hubble space telescope sends us a picture of galaxies in the universe many billions of light years distant from us and many billions of years back in time as they existed maybe only a billion years after the big bang, that the telescope can send us a similar picture when it is pointed in the opposite direction? In fact, it would send us a similar picture no matter in what direction it is pointed. In our real world, the big bang appears to be located out in space in all directions. The center of the universe where it all began appears to be located all around us in a sphere far, far away. This model explains why. Actually, our 3 space dimension model, stage 1, in Figure 1 shows that the center of the universe began at a single point which is at time $t=0$, just as our logic would tell us. If we are at the north pole in Figure 1 and look out into space at a galaxy, say, 7 billion light years away, we are not looking out along the surface of the sphere that represents the universe as it exists now. As mentioned earlier, we are actually looking back in time 7 billion years, due to the fact that it took light from the galaxy 7 billion years to reach us, and see the galaxy as it existed in a smaller universe; i.e., a smaller sphere with a radius equal to $t=7$ billion years where all the galaxies are much closer together. If we look out farther and farther into space, we are looking back into time billions of years more to a much smaller sphere where all the galaxies are much closer together until eventually, at 14 billion years, and over 16 billion light years away (based on expansion at a constant rate), the sphere becomes a point no matter in what direction we look from the north pole.

There is another way to visualize this. We mentioned earlier when we were explaining how the model can represent the changing rates of expansion of the universe, that light was first emitted in the early universe about 380 thousand years after the big bang. This time is commonly referred to as the time of recombination. We are now receiving this light as radiation, detecting it in telescopes, and calling it the Cosmic Microwave Background (CMB) radiation. It is coming from hot clumps of gas and atoms, which would become stars and galaxies, which are spread throughout a sea of hot plasma. We are receiving it from all directions from outer space. When we look at a map of the CMB, we are looking at a flat projection of the radiation coming from the inside surface of a giant sphere, where we are at the center and located many billions of light years away from the surface. The CMB was first detected over 50 years ago. What we need to realize is that the CMB we are receiving now is not 
the same CMB we received 50 years ago. The CMB we are receiving now is from matter in the early universe that was about 50 light years closer to the point at which the universe began, and 50 light years farther away from us than the CMB from 50 years ago.

We on earth, in the Milky Way galaxy, had our beginning in one of these hot clumps of gas and atoms located somewhere within this giant blob of extremely hot plasma. At the time of recombination, the universe had already expanded at such a rate that the clumps were moving apart from each other so fast that it is just now after more than 13 billion years, that light from some of these clumps is reaching us. We can visualize ourselves in this expanding blob where everything is moving away from us in all directions. The galaxies that are now closer to us were the clumps that were closer to us, and we received their first light, their CMB, billions of years ago. The CMB we are now receiving is from clumps so far away, or moving away so fast, that it has taken over 13 billion years for us to receive it. As the years go by, we will be receiving the CMB from the clumps that were even farther away from us at the time of recombination. As we go back deeper in space and farther in time, we encounter the rest of the universe that is not yet visible to us, back to the time of the big bang. Depending upon how fast the universe is expanding, we may never receive the CMB from the most distant clumps that are now galaxies 125 billion light years away from us in our model. This visualization shows how the model represents our perception of the universe as we look out into space. It shows how the big bang and the CMB can actually appear to exist in a sphere all around us, and come from all directions in space.

This 5-dimensional model (4 space dimensions and the time dimension) can be used to show another very interesting result. In Figure 8, if we look at the surface of the sphere at time, $t=0$, the big bang, the model shows us that time is proceeding inward in the universe. In the model, this means that the sphere at time, $t=0$, becomes larger as time progresses inward, and the time radius ends at the observer. In real life, this reflects the fact that time progresses from the past to the present where the observer exists. As the observer looks out into space in any direction, the observer is looking back in time and sees things as they were in the past. As the future develops into the present from the point of the observer, the timeline of the past increases, as shown in the model as the $t=0$ sphere becomes larger. As the size of the entire universe increases, all the visible matter expands outward from the observer, just as current experimental data confirms. To be more exact, this shows that the observer is moving away at an increasing rate from all the matter observed all the way back to the big bang. For example, when current data using the Doppler effect shows that a galaxy 10 billion light years away from our galaxy is moving away at a faster rate than one that is 5 billion light years away, it shows that the distance between our galaxy and a galaxy that we now observe where it was 10 billion years ago, is increasing faster than the distance between our galaxy and a galaxy we now observe where it was 5 billion years ago. If these other two galaxies still exist now, it means that the galaxies are mutually moving away from each other in a universe that is expanding at an accelerating rate. This is what our model shows.

Another comment should be made regarding the model. Can "orientation" really be a $4^{\text {th }}$ space dimension? Included in the Appendices to this paper are two examples of how and why orientation needs to be considered as a $4^{\text {th }}$ space dimension. The examples are presented as stories, similar to thought experiments that a teacher in a space dimension class delivers to his students. The first one is titled, "The Space Dimension Class", and the second one is titled, "Lost in Space”.

Also, in his book, The Elegant Universe, Brian Greene tells the story of how, in 1919, a mathematician named Theodore Kaluza suggested that there may be more than 3 space dimensions. His theory involved using equations analogous to those Albert Einstein used in his general theory of relativity, but included a $4^{\text {th }}$ space dimension and the time dimension instead of Einstein's 3 space dimensions and the time dimension. As a result, he found extra equations. He realized that the extra equations "were none other than Maxwell had written down in the 1880's for describing the electromagnetic force! By adding another space dimension, Kaluza had united Einstein's theory of gravity with Maxwell's theory of light” [5].

Brian Greene continues to relate how further detailed study of Kaluza's proposal showed that it conflicted with experimental data. "The simplest attempts to incorporate the electron into the theory predicted relations between its mass and its charge that were vastly different from their measured values” [6]. As a result, many physicists lost interest in Kaluza's idea. In the 1970's and 1980's, interest began again to focus on extra space dimensions as string theory evolved. String theory attempts to unify the fundamental forces of nature and requires up to 10 space dimensions and one time dimension. All the extra space dimensions are considered to be “curled-up” dimensions at every point in our familiar 3-dimensional space. They are required to be so microscopic that we cannot observe them. These are completely different than the proposed extra space dimension 
called orientation. As discussed thus far, orientation is a large space dimension that is equivalent to height, width, and depth, and is a dimension we observe in our daily lives. We just cannot experience more than one particular orientation at a time, whereas the 4-dimensional universe does. It would be interesting if the physicists and mathematicians could formulate equations to represent orientation as the $4^{\text {th }}$ space dimension, and see if this would correct the experimental data problems experienced by Kaluza when he had united Einstein's theory of relativity with Maxwell's theory of light. It should be noted that orientation cannot be represented mathematically nor graphically as some combination of the 3 familiar space dimensions. There has to be an additional framework upon which the 3 space dimensions of height, width, and depth are oriented to show which way is "up". It is a completely different space dimension that is required to represent or describe an object in space.

Before we discuss dark matter, it is important to emphasize that this stage 2 model was developed from the perspective of the observer, which is how we view and study our universe. We are not at the center of our universe. All the galaxies 125 billion light years away from us at the surface of the larger sphere in the model are not at the edge of the universe any more than our galaxy is at the edge of the universe from the perspective of an observer in one of those galaxies who has developed a similar model. Again, everything on the outside of the model, which represents our universe, is the future.

\section{Dark Matter}

What is dark matter? Dark matter is commonly defined as a hypothetical form of matter that is believed to make up over 90 percent of the matter in the universe but is invisible in that it does not absorb or emit light, and does not collide with atomic particles but exerts gravitational force. Originally it was the so-called "missing matter" in the universe that was needed to account for the motions of the stars in numerous galaxies observed by astronomers. Once it was discovered that all observed galaxies had up to ten times more invisible or dark matter than visible matter, it was no longer called "missing" because it had then been discovered.

There is another form of dark matter that must exist if the big bang inflation theory is correct. This theory, among other things, is used to explain how matter in galaxies now very distant from each other could have communicated with other matter before, during, or immediately after the big bang. This has reintroduced a "missing matter" form of dark matter in addition to that in and around the galaxies. The inflation theory implies that there must be up to another ten times more dark matter than visible matter in the universe, which means that very little of the universe is composed of visible matter. Much of this "missing matter" may also be in the form of dark energy, which was postulated in the late 1990's to necessarily exist to cause the acceleration of the expansion of the universe and is detectable only through its gravitational effects. As mentioned earlier, NASA Planck space mission data show that the early universe was 4.9 percent real visible matter, 26.8 percent unseen dark matter and 68.3 percent unseen dark energy.

When we compared the volume of the entire universe to the volume of the visible universe in our model, the ratio came out to be 20.7 to 1 . This means that if our visible universe contains only 4.9 percent real visible matter, then our entire universe must contain 20.7 times that, or a total of 101 percent matter that is real matter, but is not observable in our visible universe at the present time. This is an amazing result which can explain from where the dark matter and dark energy in our visible universe is coming. Since the entire universe contains 20.7 times as much real matter as there is in our visible universe, and we are calling it dark matter and dark energy, the value of 101 per cent is essentially the same as the 100 per cent total of visible matter, dark matter, and dark energy $(4.9 \%, 26.8 \%, 68.3 \%)$ reported by the NASA Planck space mission. The dark matter and dark energy in our visible universe is not matter, it is gravity from all the real matter in our entire universe. Remember, dark matter was originally introduced to account for the gravity that has to exist in galaxies to cause the rotation of the stars in the galaxies. It does not have to be matter. It can simply be gravity.

An important point to realize is that this does not contradict current theories and explanations that dark matter and dark energy cannot be real matter made of atoms (baryonic) due to other reasons, such as nucleosynthesis, and calculations showing that our universe is flat and at its critical density. The result above still agrees with the results of other calculations that real matter can only be about $4.9 \%$ of all the matter (gravity) in the universe. The 4.9\% real matter still remains in our model. The model shows that there is just 20.7 times more of it in our entire universe, which is producing 20.7 times more gravity in our entire universe (including our visible universe) than can be produced by the real visible matter in our much smaller visible universe. The percent of real matter in the entire universe is still $4.9 \%$. We can now show how the gravity that exists in our visible, observable un- 
iverse, which we now call dark matter and dark energy, is the gravity produced by all the real matter in the rest of the universe.

We first of all have to show how gravity can appear to act instantaneously throughout the universe, because an immediate argument is that we must disregard all matter outside of our visible universe because its effects or influence cannot travel faster than the speed of light. The counter argument is that all the dark matter and dark energy outside of our visible universe affects the expansion of our visible universe because it is part of the entire universe, not because its effects have to travel to the visible universe. We can show this by using one of Sir Isaac Newton's gravitational laws, and also using a modification of Alan Guth's inflation theory which will include gravity.

\section{Gravity as Dark Matter and Dark Energy}

One of Sir Isaac Newton's gravitational laws explains how the effects of gravity from all the stars and matter in a galaxy can be analyzed. For example, how do all the stars and matter in the Milky Way galaxy affect the sun in its orbit around the center of the galaxy? Donald Goldsmith, in his book, The Astronomers, presents a very good explanation of this law and how it predicts the motion of stars in galaxies [7]. The first part of Newton's law states that all the stars and matter outside the sun's orbit can be ignored, because their gravitational effect on the sun and its orbit cancels itself out. The second part states that the gravitational effect of all the stars and matter inside of the sun's orbit can be concentrated at the center. This by the way, was the law that was used to discover the dark matter that exists in a halo effect around galaxies to account for the faster than expected motion of stars in the outer orbits of the galaxies.

If we apply the second part of this law to our model in Figure 8, we can simply state that the gravitational effect from all the galaxies and matter inside the outer sphere (representing the entire universe) at a distance of 125 billion light years from the center, can be concentrated at the center. The center is where we exist as the observer in our galaxy. Any other observer in any other galaxy in the universe will likewise be at their center. This means that the gravitational effects of all the matter in the universe are spread out fairly uniformally throughout the universe. Each observer in each galaxy is feeling the concentrated effect of gravity from all the galaxies in the universe. This is how all the real matter in the entire universe, which is 20.7 times the visible matter in our visible universe, can produce the gravity in our visible universe which we are calling dark matter and dark energy.

A modification of Alan Guth's inflation theory can explain how the gravitational effects from all the galaxies in the universe can be spread throughout the universe, even though the galaxies are separated by distances much greater than light could have traveled between them. The first part of this modification relies on the fact that, in the evolution of the universe, gravity separated itself from the other forces of nature before the inflationary period of the universe began. Brian Cox, in his book, Wonders of the Universe, presents a graphic which shows the timeline of the universe from the beginning of time at the big bang, to the present [8]. It shows that the Planck Era, which is the time when gravity separated from the other forces of gravity, began at a time of $10^{-43}$ seconds. It shows that the Grand Unification Era, which is the time when the strong nuclear force splits from the other forces, began at a time of $10^{-36}$ seconds. The Inflation Era began at the end of the Grand Unification Era. It is generally agreed that the period of inflation occurred from $10^{-36}$ to $10^{-32}$ seconds. The point is, that gravity existed throughout the universe before inflation began.

The second part of the modification of Alan Guth's inflation theory introduces gravity into his original theory. Years ago, cosmologists, astronomers and astrophysicists wondered how objects (matter) in far distant reaches of the universe more than 13.8 billion light years apart (the age of the universe), could be so homogeneous and so much alike in many properties, such as composition and temperature, when there was no way that light or any type of information would have had the time to travel from one to the other. The solution was the inflation theory proposed by Alan Guth in the early 1980's, as mentioned earlier. The theory basically proposes that all of the matter in the universe, in some form, was initially close enough together to share its properties. As the big bang began and the matter expanded and clumps moved away from each other, an inflationary period took place in the expansion where the clumps, eventually to become stars and galaxies, moved away from each other much faster than the speed of light. After this rapid expansion much of the matter was separated by distances greater than the distance light could have ever traveled since the beginning of the universe. The distant regions of the universe could never again transfer information or have any influence upon each other (unless a deflationary pe- 
riod should occur).

If the inflation theory, which after some modification is now widely accepted, can explain why the properties of similar objects in distant regions of the universe can be so uniform because they moved away from each other much faster than the speed of light, should not it also be able to explain why gravity could be "left behind", so to speak, around objects that rapidly moved apart from each other faster than the speed of light? If the effects of gravity cannot travel through space faster than the speed of light, as is generally believed based on Albert Einstein's General Theory of Relativity, and the matter separated faster than the speed of light, some of the gravity had to be "left behind". And as we discussed earlier, the total amount of this gravity is 20.7 times the amount produced by the real matter in our visible universe. This can be the gravity that exists in galaxies as dark matter, and the gravity that exists throughout the universe as dark energy.

The following example may make it easier to understand how gravity can be "left behind". Let us suppose that the early universe was similar to a very massive soccer ball with all the mass concentrated in the outer shell of the soccer ball, similar to our stage 1 model. There are indentations or seams in the soccer ball similar to a regular soccer ball, which usually has 20 hexagonal and 12 pentagonal sections. The shell of the soccer ball is a curved surface and all the regular hexagonal and pentagonal sections inside the seams of the soccer ball are likewise curved surfaces. See Figure 9.

The size of the soccer ball determines the amount of curvature of the soccer ball as a whole, and also the curvature of each individual section of the soccer ball. For simplicity, let us now look at a cross-section of the soccer ball. See Figure 10.

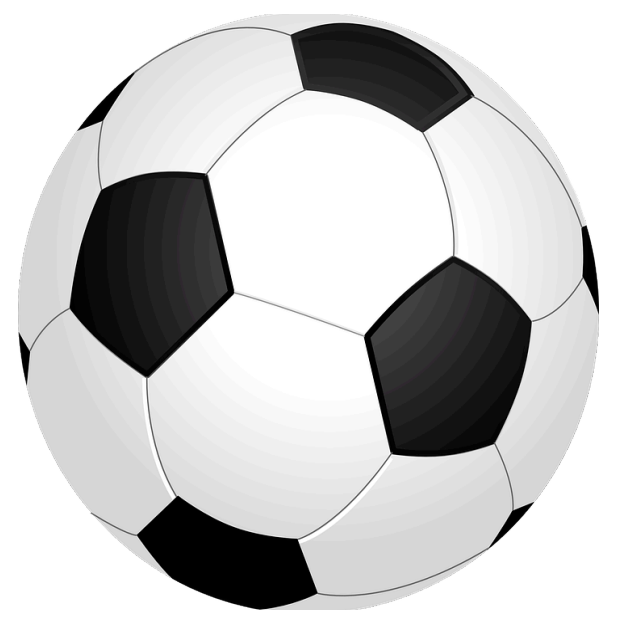

Figure 9. Soccer ball.

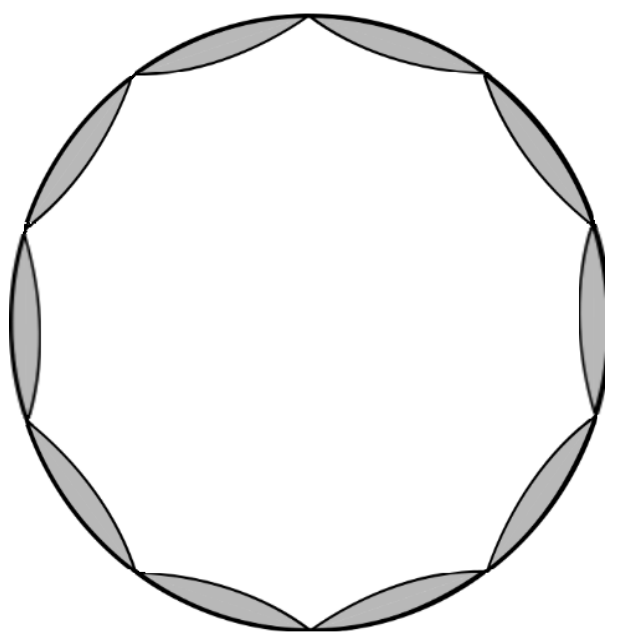

Figure 10. Soccer ball cross-section. 
Suppose the soccer ball undergoes a large expansion, and the seams or indentations are very thin and much weaker, but much more elastic than the material inside the seams. The material inside the seams is very strong and thick and not very flexible. As the soccer ball expands, the space between the hexagonal and pentagonal sections stretches so that the sections are now spaced 5 times further apart than they were before the expansion. Because of their thickness and strength, they are still approximately the same size and have about the same curvature as before the expansion. The radius of the soccer ball will now be 5 times as large, and the curvature of the cross-section of the soccer ball as a whole, which is a circle, will now be 5 times less than before the expansion (since it is inversely proportional to the radius), but the curvature of each hexagonal and pentagonal section will be approximately the same as before the expansion. See Figure 11.

Let us now make the analogy between the soccer ball and our universe, but assume the universe has many, many more sections than the soccer ball since it is much, much larger. It follows that if the expansion of space occurs in the seams between the sections (space between the galaxies), the curvature (warped space-time) within the galaxies will remain about the same while the curvature (warped space-time) of the universe as a whole will be 5 times smaller (because the radius of the cross-section of the universe is now 5 times larger). Gravity, which is the force of attraction between the galaxies, and is also the warped space-time in and immediately around the galaxies, has been "left behind" in and around the galaxies as space has expanded between the galaxies. When we now look at the expanded universe where the galaxies are now 5 times farther apart then before, and calculate the amount of mass we can observe in the galaxies, it will be much less than is needed to account for the gravity (warped space-time) in and around the galaxies. Thus, the dark matter that is needed to exist in a spherical halo around the galaxies to account for the motions of the stars observed by our astronomers within the galaxies can be the gravity produced by all the galaxies when they were much closer together, that was "left behind" when the inflation occurred.

\section{Dark Energy}

Dark energy is commonly defined as an unknown form of energy which permeates all of space, and tends to accelerate the expansion of the universe. The NASA Planck space mission shows that it makes up $68.3 \%$ of the universe. It is important to note that, although the Planck space mission collected its data from a detailed study of the Cosmic Microwave Background (CMB) radiation, the values of $4.9 \%, 26.8 \%$, and $68.3 \%$ are the present day values extrapolated from the CMB data. They are not the percentages that existed when the CMB radiation was emitted from a 380,000 thousand year old universe. Current thought is that dark energy is a negative pressure spread throughout space as a part of space itself, which acts as an anti-gravity force. As space expands, the dark energy is not diluted. Instead, more dark energy is created as space is created. However, this is not how energy, in general, behaves.

Our stage 2 model offers an alternative explanation. We can show with the model, that instead of dark energy

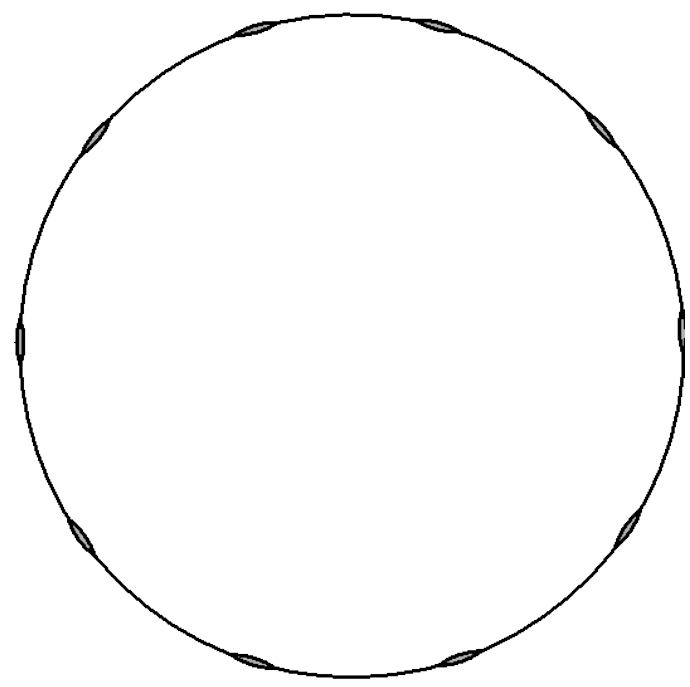

Figure 11. Soccer ball expanded. 
increasing to cause the accelerated expansion of the universe, the total amount of matter in the universe is decreasing to cause the amount of gravity holding the universe together to decrease and allow the increased expansion.

Here is the theory. We know from physics that the amount of energy in a certain volume is diluted as the volume increases. The volume of a given amount of space increases by the cube of the radius as it increases, $\left(V=\frac{4}{3} \pi r^{3}\right)$. We also know from physics that the amount of gravity holding a given amount of space together decreases as the radius increases by square of the radius, $\left(G=\frac{1}{r^{2}}\right)$. Thus, if we look at our stage 2 model, the energy spread throughout the volume is decreasing faster (by the cube of the radius) than the gravity holding it together is decreasing (by the square of the radius). This means that the expansion should be slowing down. However, if the amount of gravity is reduced by conversion of matter into energy by the equation, $E=m c^{2}$, greater than the volume increases, the space will expand. We can use the model to calculate the numbers that will show this.

How is the amount of matter (gravity) being decreased? There are many books and articles written about the evolution of the universe, and how stars are formed, live, and die. We now have stars that are several generations old. The heavier elements in our universe are created in the life cycles of the stars. In the life cycle of a star, matter is continually being transformed into energy through the fusion process. When the life cycle of most stars ends in a supernova explosion, tremendous amounts of energy are released as heavier elements are created, and the amount of matter, although heavier, is much less. Since stars started forming in the universe about 13.3 billion years ago, the total amount of matter in the universe has been decreasing. We can calculate how much matter has been transformed into energy every billion years to cause the expansion of the universe to accelerate about 6 billion years ago.

We first of all, have to have an estimate of the number and type of stars that exist in the universe, and we also have to have an estimate of how long the life cycle of the average star lasts. The astronomers, astrophysicists, and cosmologists who have studied the stars have come up with some detailed results. They have learned that the greater a star's mass is when it formed, the faster it burns its fuel, and the shorter is its life cycle, which may be numbered in only millions of years. Many more stars, like the sun, will continue burning their fuel, and decreasing their mass, for about 10 billion years. The much smaller stars will burn their fuel much slower and may continue to do so for over 100 billion years.

The Milky Way galaxy is estimated to contain between 200 - 400 billion stars, and there are estimated to be over 100 billion galaxies in the universe. Each of these stars is converting tremendous amounts of matter into energy every second of every day. It is also estimated that the life cycle of the average star in the Milky Way galaxy is about 50 billion years. Astronomers can determine the age of stars in the universe very accurately. They have estimated that some of the stars in the Milky Way galaxy have been in existence for over 13 billion years, thus, the Milky Way galaxy must have been one of the earliest galaxies to form within the first billion years after the beginning of the universe.

We can now calculate how much matter has been transformed into energy every billion years to cause the expansion to accelerate about 6 billion years ago when the universe was almost 8 billion years old. When the expansion of the universe began to accelerate at about 8 billion years after its beginning, most of the stars in the Milky Way galaxy had already converted about 8 billion years worth of their 50 billion years of fuel, or about $16 \%$ of their matter, into energy. If the Milky Way galaxy is an average size galaxy in the universe (estimates are that it is somewhat smaller than average), then the amount of matter in the universe has decreased by at least $16 \%$ when the expansion of the universe began to accelerate.

In making the calculation, we need to realize that when the expansion of the universe began to accelerate, whatever was causing it was also accelerating. If it is in fact caused by the decreasing amount of matter in the universe, then the matter has to be decreasing at an accelerating rate. This is exactly what has been happening in our universe, when the initial dust and gas and clumps of matter in the early universe began to collapse and form the early stars and galaxies. As the stars and galaxies increased in numbers into the billions, they increasingly converted matter into energy, and the amount of matter in the universe decreased at an accelerating rate.

If the amount of decrease in matter during the first billion years was only $1 / 2$ of $1 \%$, and it increased by only $1 / 2$ of $1 \%$ every billion years, it would be sufficient to account for the accelerating expansion of the universe 
when it was almost 8 billion years old. This is when the rate of increase in volume of the universe (and corresponding decrease in energy) was less than the decrease of gravity holding it together. The ratio of the change in volume to the change in gravity, which had been much greater than one, was gradually decreasing as the matter in the universe decreased causing the decrease in gravity. Between 7 and 8 billion years, the ratio became less than one, and the expansion began to accelerate because gravity decreased at a greater rate than the amount of energy in the universe decreased. The following tables show how the changes (increase) in volume of the expanding universe (decrease in energy) compare to the changes in gravity (decrease) as the universe expanded over its almost 14 billion years of existence.

Table 4 shows how the rate of change in volume of the universe changed as the rate of change in the radius of the universe stopped slowing down and began to accelerate. After decreasing for about 8 billion years, it began to increase at an accelerating rate.

Table 5 shows how the amount of gravity in the universe decreased not only as the radius increased, but also as the amount of matter in the universe decreased. After about 8 billion years, the rate of decrease began to accelerate. This is caused by the increased acceleration in the loss of matter in the universe during the past 6 billion years (an additional increase of $1 / 2 \%$ every billion years) as shown in the Mass decrease column in the table.

Table 6 shows how the change (increase) in volume became less than the change (decrease) in gravity after 7 to 8 billion years; i.e., ratio less than one, thus causing the expansion of the universe to accelerate rather than slow down as it had been doing.

These are the numbers that show how dark energy, which is causing the expansion of the universe, does not have to be some kind of increasing energy spread throughout the universe, but rather, can be a decreasing amount of gravity which can no longer prevent the expansion from accelerating. Whatever energy that existed at the big bang to cause the initial expansion can be the energy that is causing its acceleration due to the decrease of matter and gravity. These numbers have been developed from the recent data obtained by telescope and space missions that have been analyzing and studying the stars and galaxies in the universe. The astronomers, astrophysicists, and cosmologists, should be able to show how realistic the numbers are in determining the amount of

Table 4. Change in volume.

\begin{tabular}{|c|c|c|c|c|c|}
\hline Time, t & Radius, r & Volume, $\quad V=\frac{4}{3} \pi r^{3}$ & $\begin{array}{l}\text { Difference in } \\
\text { volume }\end{array}$ & Change in volume & \% Change \\
\hline 14 & 40.0 & 267,947 & 48,927 & 0.2234 & 22.3 \\
\hline 13 & 37.4 & 219,020 & 33,292 & 0.1793 & 17.9 \\
\hline 12 & 35.4 & 185,728 & 22,623 & 0.1387 & 13.9 \\
\hline 11 & 33.9 & 163,105 & 16,715 & 0.1142 & 11.4 \\
\hline 10 & 32.7 & 146,390 & 13,024 & 0.0977 & 9.8 \\
\hline 9 & 31.7 & 133,366 & 11,040 & 0.0903 & 9.0 \\
\hline 8 & 30.8 & 122,326 & 9850 & 0.0876 & 8.8 \\
\hline 7 & 29.95 & 112,476 & 9307 & 0.0902 & 9.0 \\
\hline 6 & 29.1 & 103,169 & 10,275 & 0.1106 & 11.1 \\
\hline 5 & 28.1 & 92,894 & 10,488 & 0.1273 & 12.7 \\
\hline 4 & 27.0 & 82,406 & 12,165 & 0.1732 & 17.3 \\
\hline 3 & 256 & 70,241 & 13,799 & 0.2445 & 24.5 \\
\hline 2 & 70,241 & 13,799 & 0.2445 & 0.3951 & 39.5 \\
\hline 1 & 23.8 & 56,442 & 15,984 & 76 & 7600 \\
\hline 0.5 & 21.3 & 40,458 & 39,935 & 523 & 52,300 \\
\hline 0.00038 & 5.0 & 523 & 523 & N/A & N/A \\
\hline 0 & 0 & & & & \\
\hline
\end{tabular}


Table 5. Change in gravity.

\begin{tabular}{cccccccc}
\hline Time, $\mathrm{t}$ & Radius, $\mathrm{r}$ & Gravity, $\frac{1}{r^{2}}$ & $\begin{array}{c}\text { Difference in } \\
\text { gravity }\end{array}$ & $\begin{array}{c}\text { Gravity } \\
\text { decrease }\end{array}$ & $\begin{array}{c}\text { Mass } \\
\text { decrease }\end{array}$ & $\begin{array}{c}\text { Total } \\
\text { decrease }\end{array}$ & \% Change \\
\hline 14 & 40.0 & 0.000625 & 0.000090 & 0.12587 & 0.175 & 0.30087 & 30.1 \\
13 & 37.4 & 0.000715 & 0.000083 & 0.10401 & 0.140 & 0.24401 & 24.4 \\
12 & 35.4 & 0.000798 & 0.000072 & 0.08276 & 0.110 & 0.19276 & 19.3 \\
11 & 33.9 & 0.000870 & 0.000065 & 0.06952 & 0.085 & 0.15452 & 15.5 \\
10 & 32.7 & 0.000935 & 0.000060 & 0.06030 & 0.065 & 0.12530 & 12.5 \\
9 & 31.7 & 0.000995 & 0.000066 & 0.06221 & 0.050 & 0.11221 & 11.2 \\
8 & 30.7 & 0.001061 & 0.000054 & 0.04843 & 0.040 & 0.08843 & 8.8 \\
7 & 29.95 & 0.001115 & 0.000066 & 0.05588 & 0.035 & 0.09088 & 9.1 \\
6 & 29.1 & 0.001181 & 0.000085 & 0.06714 & 0.030 & 0.09714 & 9.7 \\
5 & 28.1 & 0.001266 & 0.000106 & 0.07726 & 0.025 & 0.10226 & 10.2 \\
4 & 27.0 & 0.001372 & 0.000154 & 0.10092 & 0.020 & 0.12092 & 12.1 \\
3 & 25.6 & 0.001526 & 0.000239 & 0.13541 & 0.015 & 0.15041 & 15.0 \\
0.5 & 23.8 & 0.001765 & 0.000439 & 0.19918 & 0.010 & 0.20918 & 20.9 \\
0 & 21.3 & 0.002204 & 0.037796 & 0.94490 & 0.005 & 0.94990 & 95.0 \\
& 5.0 & 0.040000 & 4.898272 & 0.99190 & 0.0025 & 0.99440 & N/A \\
\end{tabular}

Table 6. Ratio of change in volume to change in gravity.

\begin{tabular}{ccccc}
\hline Time, $\mathrm{t}$ & Radius, $\mathrm{r}$ & Change in volume & Change in gravity & Ratio \\
\hline 14 & 40.0 & 0.2234 & 0.30087 & 0.74 \\
13 & 37.4 & 0.1793 & 0.24401 & 0.73 \\
12 & 35.4 & 0.1387 & 0.19276 & 0.72 \\
11 & 33.9 & 0.1142 & 0.15452 & 0.74 \\
10 & 32.7 & 0.0977 & 0.12530 & 0.78 \\
9 & 31.7 & 0.0903 & 0.11221 & 0.80 \\
8 & 30.7 & 0.0876 & 0.08843 & 0.99 \\
7 & 29.95 & 0.0902 & 0.09088 & 1.14 \\
6 & 29.1 & 0.1106 & 0.09714 & 1.24 \\
5 & 28.1 & 0.1273 & 0.10226 & 1.43 \\
4 & 27.0 & 0.1732 & 0.12092 & 1.63 \\
2 & 25.6 & 0.2445 & 0.15041 & 1.89 \\
0.5 & 23.8 & 0.3951 & 0.20918 & 80 \\
\hline
\end{tabular}


matter that stars, and galaxies, are converting into energy during their life cycles. It is not so much a matter of the stars creating a tremendous amount of energy during the fusion process, which may or may not be significant, but more a matter of the loss of matter. The amounts shown in the above tables appear to be in agreement with what can be extrapolated from the current literature. The tables were also developed from our model of the universe. They show how our model of the universe can explain dark energy.

\section{Conclusions}

We have developed a model of the universe that can explain dark matter, dark energy, and the fourth space dimension. The model was developed by incorporating time into geometry by using time as a radius of an expanding sphere whose surface represented our entire universe. In the stage 1 model, all of space exists on the surface of the sphere, past history exists inside the sphere, and the future exists outside the sphere. The model shows that when we look out into space in any direction, we are looking back in time to the same point in space at the origin of our universe almost 14 billion years ago. It shows how light has been traveling through the expanding space of our universe for over 13.4 billion years. It shows how we can depict both our visible universe and our entire universe as they exist at the present time.

The stage 2 model was developed by rotating the surfaces of the stage 1 model in all directions about the observer. This was done because only 2 lateral space dimensions can be shown on a surface. For an observer to experience the third space dimension, the surface had to be rotated through a volume about the observer. By doing this, we developed a fourth space dimension which we called orientation, which also incorporated time into space. It is shown that to locate and describe a point in space, 4 space dimensions are required. Height, width, depth, and orientation are required. However, we are 3-dimensional beings living in a 4-dimensional world. We can experience only one orientation at a time, just as a flat 2-dimensional being can experience only one particular height dimension at a time.

The stage 2 model presents us a geometrical framework upon which we can calculate the volume (energy) and the amount of gravity that existed in our universe throughout its history. It thus presents us with a way to explain dark matter as the gravity produced by the real (baryonic) matter that exists throughout our entire universe. And it presents us with a way to explain dark energy as the energy that caused the original expansion of the universe at the big bang that is now accelerating due to the loss of matter and gravity in the universe.

This model of our universe represents a dramatic shift in how we view our universe. It shows how you can not only visualize a 3-dimensional space and one time dimension universe in a model on a flat piece of paper, but can also visualize a 4-dimensional space and one time dimension universe in a model on a piece of paper. The model gives us new insights into the nature of our universe, and presents a framework upon which theories can be developed to explain dark matter, dark energy, and the fourth space dimension. As we continue to explore our universe, and study and analyze the stars and galaxies, we can produce the experimental data that can show that this model can indeed explain dark matter, dark energy, and the fourth space dimension.

\section{References}

[1] Davies, P. (2006) The Goldilocks Enigma. Houghton Mifflin, New York, 56.

[2] Hawking, S. (1988) A Brief History of Time. Bantam, New York, 45. http://dx.doi.org/10.1063/1.2811637

[3] Rothstein, D. (2003) Is the Universe Expanding Faster than the Speed of Light? http://curious.astro.cornell.edu/the-universe/cosmology-and-the-big-bang/104-the-universe//expansion-of-the-universe/ 616-is-the-universe-expanding-faster-than-the-speed-of-light?

[4] Rostomian, Z. (2013) The Great Space Coaster. http://www.sdss3.org/press/lyabao.php

[5] Greene, B. (1999) The Elegant Universe. Norton, New York, 196.

[6] Greene, B. (1999) The Elegant Universe. Norton, New York, 197.

[7] Donald Goldsmith, D. (1991) The Astronomers. St. Martin’s Press, New York, 37

[8] Cox, B. and Cohen, A. (2011) Wonders of the Universe. Harper Collins, New York, 110-111. 


\section{Appendix A: Light Traveling through Expanding Space}

How is light affected by expanding space as light travels through it? We know that the speed of light is a constant. It will travel, say, a distance of one billion light years in a time of one billion years. If, for example, there were 2 galaxies that were 1 billion light years apart, and over a time of 1 billion years, space has expanded by 10 percent so that they are now 1.1 billion light years apart, how far will light have traveled from one galaxy to the other during the 1 billion years?

We first of all, have to make the distinction that the two galaxies have not moved apart relative to each other due to their motion; they have moved apart due to space expanding between them. And secondly, space, or spacetime to be more accurate, is something upon which measurements can be made as it expands. Using the above example, this means that after $1 / 2$ billion years, we can locate a point in space between the two galaxies that will be midway between them, or 0.525 billion light years away from both of them. We can then realize that light, traveling for $1 / 2$ billion years, will not have reached the half-way point of expanded space, or 0.525 billion light years. This is because the distance it had to travel was continually increasing as light traveled through it, and the last half of its journey, 0.525 billion light years, is still ahead of it, and will be continually expanding as light travels through it.

We can use the following analogy to make this clearer. Suppose we have an automobile traveling at 100 miles per hour on a roadway that is expanding by 10 percent, or a distance of 10 miles, every hour. There are mile markers every mile on the side of the road which is not expanding, (the base grid), and there are mile markers every mile on the expanding roadway. We can show how the automobile travels along the roadway in a time of 1 hour by the following graph. For simplification, the grid line markers on the base grid are shown every 5 miles, and the markers on the expanding roadway are shown every 25 miles. See Figure A1.

The graph shows that when the automobile travels on the roadway for the first 15 minutes, the length of the roadway is increasing ahead of it (the first 25 mile section will increase by $1 / 4$ of the distance the 100 mile roadway increases in 15 minutes, or $1 / 4(2.5)=0.625$ miles) so that it will not reach the 25 mile marker on the roadway. When it has traveled, say, $1 / 2$ the 25 miles (in 7.5 minutes), the remaining half of the 25 mile roadway will have increased by $1 / 2$ of 0.3125 miles, so the automobile will be at least that much short of the 25 mile marker. As it travels the remaining half of the 25 mile roadway, the roadway continues to expand by the same amount, so the automobile will end up about 0.3125 miles short of the 25 mile marker. As the automobile continues to travel on each additional 25 mile expanding section, it will continue to fall short of each section mile marker by an increasing amount. But, note that the sections it has already traveled over also continue to expand, by 0.625 miles per 25 mile section, which will increase the distance the automobile has traveled in reference to the base grid. This is also shown in Table A1.

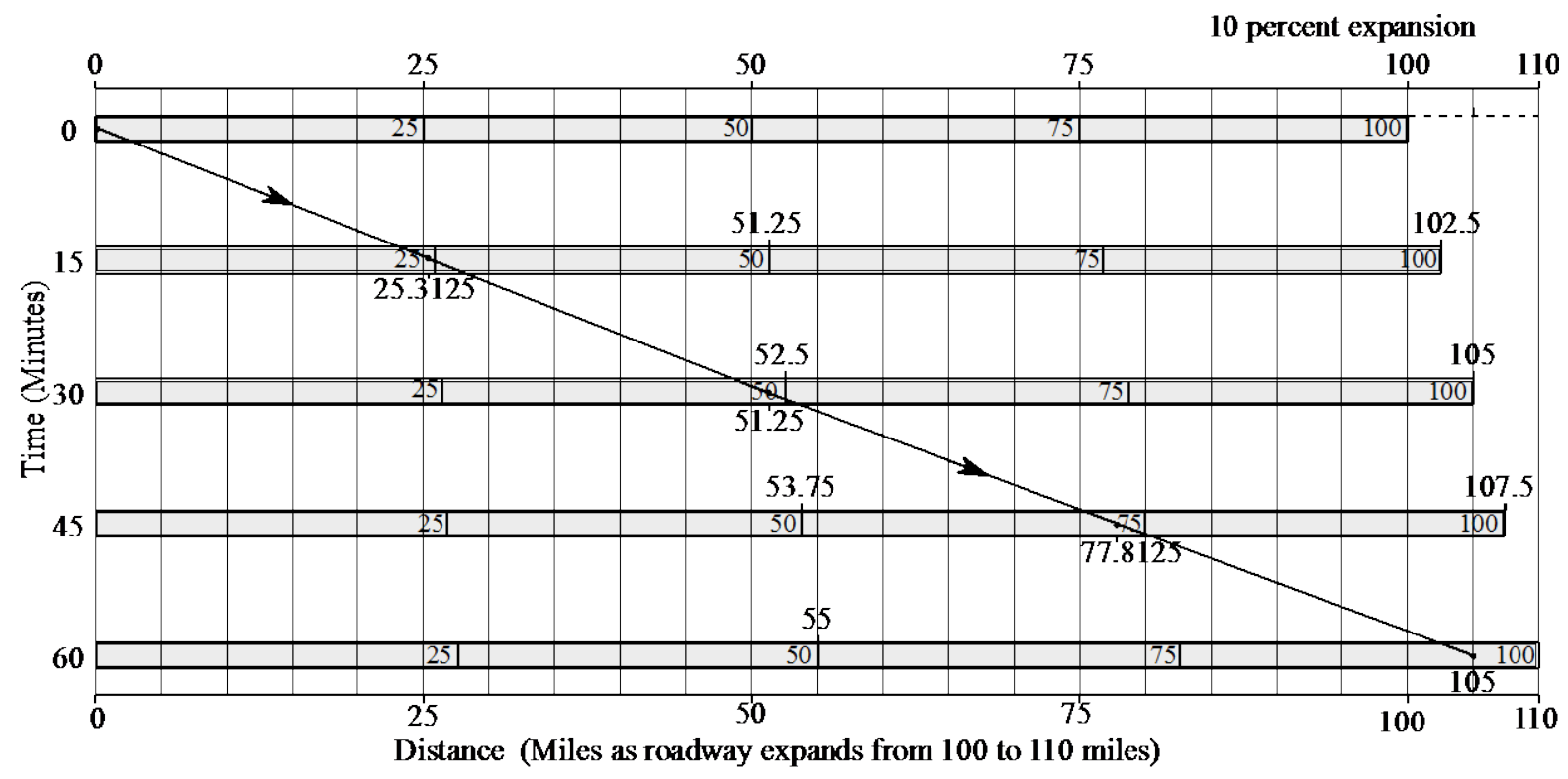

Figure A1. Analogy of an automobile traveling at $100 \mathrm{mph}$ on an expanding roadway. 
Table A1. Distance automobile travels in reference to base grid.

\begin{tabular}{|c|c|c|c|c|c|}
\hline \multirow{2}{*}{$\begin{array}{c}\text { Time } \\
\text { (minutes) }\end{array}$} & \multicolumn{5}{|c|}{ Distance traveled on original roadway as it expands (miles) } \\
\hline & $0-25$ & $25-50$ & $50-75$ & $75-100$ & Total \\
\hline 0 & 0 & & & & 0 \\
\hline 15 & 25.3125 & & & & 25.3125 \\
\hline 30 & $25.3125+0.625$ & 25.3125 & & & 51.25 \\
\hline 45 & $25.3125+2(.625)$ & $25.3125+0.625$ & 25.3125 & & 77.8125 \\
\hline 60 & $25.3125+3(.625)$ & $25.3125+2(0.625)$ & $25.3125+0.625$ & 25.3125 & 105 \\
\hline
\end{tabular}

The end result is that the automobile will have traveled a total of 105 miles on the roadway as it expanded from 100 to 110 miles in length. It has traveled 1/2 the expanded distance.

By analogy then, we can say that when light travels through expanding space, it will travel about $1 / 2$ the distance by which space has expanded during the same time frame. This assumes, as mentioned earlier, that space, or spacetime, is actually something which can expand, and does expand. Albert Einstein assumed that spacetime was something that could be warped when he theorized about gravity. Astronomers have proven him to be correct when they have seen in pictures produced by the Hubble space telescope, that light is indeed bent by warped spacetime as it travels around massive galaxies in a lensing effect. The big bang theory assumes that space expanded enormously at the beginning of the universe, slowed down, and is now expanding at an accelerating rate. Thus, I believe we can state that when light travels through expanding space, it will travel $1 / 2$ the expanded distance.

\section{Appendix B: The Space Dimension Class}

Suppose you were educated in a school system where it was required that you pass a space dimension class before you could graduate from high school. A prerequisite for the class was that you complete basic mathematics classes to include fractions, and complete basic geometry classes to include formulas for determining the area and volume of simple shapes and solid objects such as cubes and spheres. The space dimension class was a 2-hour class and consisted of a 1-hour lecture by the space dimension teacher followed by a 1-hour exam. If you failed the course, you would have to re-take it until you passed it, but if you had to re-take it, it would be a slightly different, more complicated course.

You are now one of 24 students in your first space dimension class. The space dimension teacher is standing behind his desk at the front of the classroom with a large chalkboard on the wall behind him. He begins his lecture and talks about the space dimension called height, which he explains is a length in the up and down direction. He then explains how the space dimension called width is a length in the left and right direction, and the space dimension called depth is a length in the forward and backward, or front and back direction. He also explains how these particular dimensions are based on a Cartesian coordinate system, which is also called a rectangular coordinate system, but there are also other possible coordinate systems, such as a cylindrical coordinate system that uses 2 lengths and 1 angle to locate a point in space or describe an object, or a spherical coordinate system that uses a length and 2 angles to locate a point in space or describe an object. He also mentions that there is a polar space coordinate system that uses a combination of the rectangular coordinate system and 1 length and 3 angles to locate a point in space. "However," he explains, "regardless of the coordinate system used, it is generally recognized that there are only 3 distinct space dimensions, which should be fairly obvious, because any object you can see and touch and feel appears to have height, width and depth”.

He then reaches into a large, 5-gallon bucket sitting on his desk and pulls out a white Styrofoam cube about the size of his fist. He then explains how you could remove the top half of the cube by passing a horizontal plane through the middle of the cube. He also explains how you could remove the left half of the cube by passing a vertical plane through the original cube to separate the left half from the right half, and also how you could remove the front half of the cube by passing another vertical plane through the original cube to separate the front half from the back half. He then goes on to explain how you could remove only the top half of the left half of the 
original cube by cutting along a portion of the horizontal and vertical planes so that the top left quarter (1/4) of the cube was removed (which is $1 / 2$ times $1 / 2$ equals $1 / 4$ of the cube). To visually emphasize this, he picks up a razor blade from his desk and proceeds to make two cuts into the cube and removes the top left quarter of the cube, which is the top half of the left half of the cube. See Figure B1.

He places this cube on his desk and reaches into the large bucket and pulls out a second Styrofoam cube the same size as the first. He now explains how to remove the left third (1/3) of the top half of the front half of the cube. The part that would be removed would be $1 / 3$ times $1 / 2$ times $1 / 2$ equals $1 / 12$ of the cube. Again, he takes a razor blade and proceeds to make three cuts into the cube and removes the corner of the cube corresponding to the left third of the top half of the front half of the cube. See Figure B2.

The space dimension teacher places this cube on his desk and then tells all the students that the lecture is now over, and that it is time for them to take the final exam. He tells them that in order to pass the space dimension class, they will have to complete one problem. He then turns around, writes the problem on the chalkboard, and then walks out of the classroom. Here is what he writes on the chalkboard:

"Final Exam Problem:

Come up to my desk, reach into the bucket and remove one cube. Take the cube and one of the razor blades on my desk to your desk and remove the front half of the right half of the top half of the cube. You have one hour. Then take the remaining part of the cube to my office, place it on my desk, and compare it to my solution,

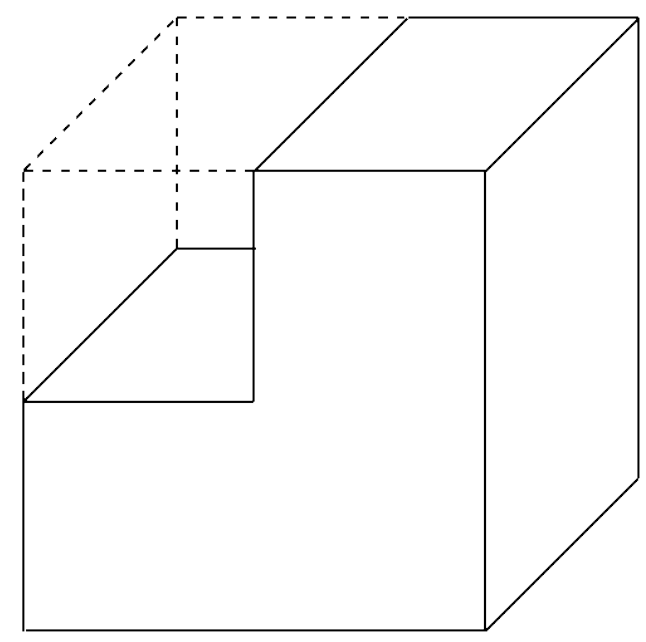

Figure B1. The top half of the left half removed.

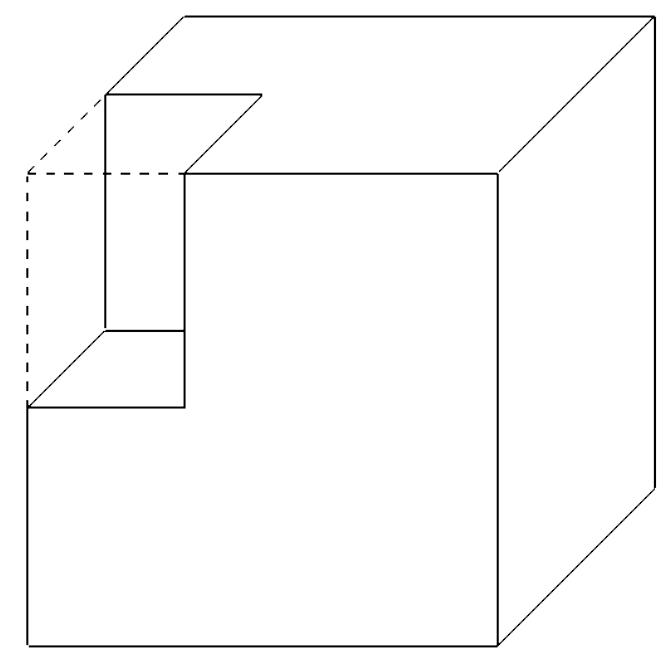

Figure B2. The left third of the top half of the front half removed. 
which will be sitting in the middle of my desk. My original cube is identical to your original cube. If your cube looks the same as mine after you remove the specified section, you pass the course."

At this point, you think, "Hey, this is fairly easy!” since the lecture was not that difficult. You are very impressed by the space dimension teacher. He has very thick white hair, so he has probably been doing this for a long time. He appears to be very intelligent, and explains things in a very detailed, methodical manner. Anyway, you go up to the space dimension teacher's desk, reach into the bucket, and pick up a cube. When you look at the cube, you see that each of the 6 sides of the cube is a different color! One side is red, one side is black, another side is green, the fourth is blue, the fifth is yellow, and the sixth is white. All of the other students pick out an identically colored cube. Your heart starts to sink because you now realize that you have to decide which side of the cube is "up". The space dimension teacher will have obviously picked one of the colored sides of the cube to be in the "up" position when he made his 3 cuts into the cube to remove the top, right, front section of the cube. The orientation of your cube when you make the razor blade cuts, must be the same as his if your solution is to be the same as his. Unfortunately, you did not have the chance to ask the space dimension teacher which side of the cube was "up" since you assumed all sides of the white Styrofoam cube could be treated equally. Even if you had thought of the question, you did not have the opportunity to ask it.

You return to your desk, and after considerable thought, which leads you nowhere, arbitrarily pick the red side to be in the "up" position, and the blue side to be the "front" position, and make the 3 cuts to remove the top, right, front $1 / 8$ section of the cube. At the end of the hour you take your cube to your space dimension teacher's office and place it on his desk next to his cube. You feel elated as you see that you chose the correct "up" side! Both your cube and the teacher's cube have the red side placed in the "up" direction! Furthermore, the top, right, front section that was removed on your cube corresponds to the same top, right, front section on the teacher's cube! But your heart sinks as you notice that the blue front side of your cube is not the same as the green front side of your space dimension teacher's cube. You flunked the class! See Figure B3.

Your fellow classmates go through the same experience that you just went through, and then you all return to the classroom with the space dimension teacher. He tells the class that only 1 student passed the course, which is exactly what he had expected, because each of the 24 students had only a 1 in 6 chance of arbitrarily picking the red side of the 6-sided cube to be the "up" side. In addition, of the 4 students that correctly picked the "up" side, each had only a 1 in 4 chance of picking the correct "front" side out of the 4 remaining vertical sides of the cube. He tells the students that according to custom, he will place the name of the student on the cube that was the same as his, and place it in the trophy case in his office to save for years to come. All other cubes will be discarded. He then tells the class that he hopes that all the students now realize the importance of this $4^{\text {th }}$ space dimension called "orientation". To emphasize his point, he asks all the students to place themselves in the following situation:

"Suppose”, he says, "you were a very tiny, though very intelligent microbe living inside one of the Styrofoam cubes and were able to move freely, although slowly about the cube. You could actually move to the surface of the cube, poke your head out, and see what color the surface happened to be. You had been living in your cube universe that was inside the 5-gallon bucket all your life. You had actually traveled up to the top of your universe to see which side was 'up'. You knew which side was 'up' because you felt the pull of gravity and could

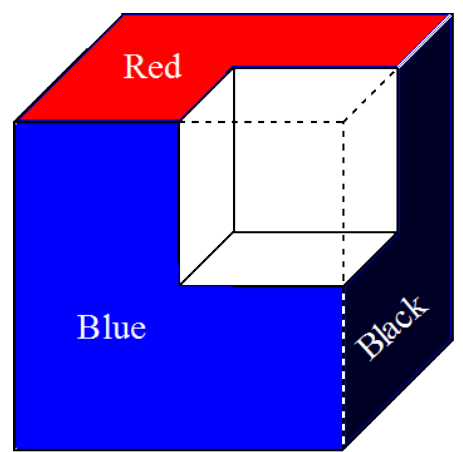

The student's cube

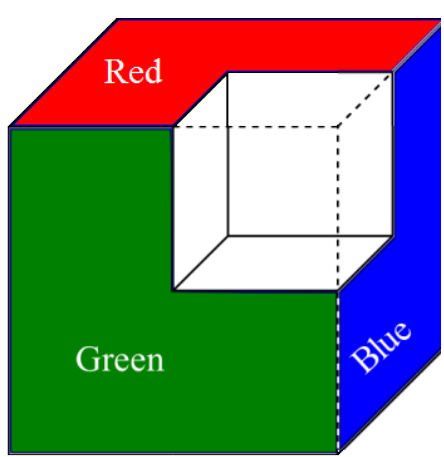

The teacher's cube

Figure B3. The student's and teacher's cube. 
orient yourself so that 'up' was in the direction of the top of your head. You discovered that no side was truly 'up'. Because of the way your cube was positioned in the bucket, 'up' turned out to be a corner of the cube with a yellow side, a white side, and a green side each sloping downward at slightly different angles from the corner. That was fine with you because your entire universe was made of Styrofoam, and the color and shape of the outside had no influence upon your life. You were very comfortable living near the center of the cube, and you thus proceeded to go about your life as usual.”

"You now suddenly feel your universe being moved about, turned upside down, and turned around, and then finally come to rest as a student places your universe on his desk. You hear someone muttering something about removing the top, right, front section of the cube within an hour and discarding it. You have a 1 in 8 chance of being removed from your universe and discarded, depending upon where you are located inside the cube. You know which side is now 'up', even though it is now much different than it was several minutes ago, because you can feel the pull of gravity pulling at your left ear. You quickly re-orient yourself inside your universe so that the pull of gravity is now at your feet and 'up' is now at the top of your head. You still have a major problem in that you have no idea which direction you should face so that your 'front' side corresponds to the student's 'front' side. However, since you are a very intelligent microbe, you quickly figure out that all you have to do is move down into the bottom half of the cube and you will have a 100\% chance of surviving inside of your universe, provided your student understood the lecture about the 3 space dimensions. You don't know where the right side of the cube is, part of which will be removed; and you don't know where the front side of the cube is, part of which will also be removed; but you do know where the bottom half of the cube is, none of which will be removed. You start moving downward in your Styrofoam universe as quickly as possible, which unfortunately is slower than a snail's pace. Fortunately though, you make it into the bottom half just in time as the top, right, front of your universe disappears. You breathe a huge sigh of relief and thank your lucky stars that you knew all about the 3 space dimensions involving up and down, left and right, and front and back, and the other space dimension called orientation. You are also very thankful for the something in your life called gravity, which enabled you to determine which orientation was 'up'.”

"You now feel yourself being picked up, carried around, and placed down on a surface again, and the pull of gravity is still at your feet. Your universe has just been placed on the space dimension teacher's desk, and unfortunately for you, the student that had your cube picked the white side of the cube to be the 'up' side instead of the red side, which was the teacher's determination of the correct 'up' side. Your whole universe will now be discarded because neither you nor the student knew what the correct orientation of the cube should be to agree with that of the authoritative space dimension teacher."

The space dimension teacher pauses for a bit and then tells the students how fortunate they all are that they are not tiny microbes living in a Styrofoam universe. He tells them that he hopes they have learned something worthwhile in his class, and is looking forward to seeing them again when they re-take the class. He tells them they will learn more about the dimension called orientation in the next class. He also invites the one student who passed the course to also re-take the class since it was only by chance that the student passed, not because of the student's superior understanding of the space dimensions. He then dismisses the class, wishing them well in their future endeavors.

This little story will hopefully cause you to think about the possibility of a fourth space dimension actually existing in our universe, and how it possibly could be something called orientation, but a kind of orientation that will be considered in a slightly different context than that which we now experience.

\section{Appendix C: Lost in Space}

Can "orientation" really be a $4^{\text {th }}$ space dimension? We should take time to visit another of the space dimension teacher's classes. One of his advanced classes is called "Orientation: The $4^{\text {th }}$ Dimension". In this class, he always begins with a little story. "Suppose," he says, "you were in a very large spaceship far out in space conducting a mission of exploring the universe, and had just recently entered a very unique-looking interstellar gas and dust cloud. The dust was not all that visible, but it quickly became thick enough so that your visibility was limited to several hundred miles in any direction, and neither stars nor any other objects were visible. Suppose the commander of the spaceship brought his spaceship to a stationary position and then asked you to board one of the spaceship's small personal reconnaissance vehicles and make an exploratory trip to see how distant the gas and dust cloud extended. To maintain direction and know where you were at all times, you were to keep de- 
tailed notes as you left the exit port at the rear of the spaceship. You were to keep track of exactly how many miles you traveled in each of the 3 space dimensions; i.e., forward/backward, left/right, and up/down, so that you would be able to retrace your travel and return easily."

"Suppose", he continues, "that during your trip, a burst of interstellar radiation suddenly occurred, and as it passed through your location, it caused your reconnaissance vehicle to spin and tumble about and disabled your gyroscope and propulsion and navigation systems. However, you had kept careful notes, and you knew that at this point, you had traveled a net of 650 miles in the forward direction, a net of 210 miles in the left direction, and a net of 360 miles in the upward direction since you had left the spaceship. Fortunately, although you had lost sight of the large spaceship several hundred miles ago, you still had a communication capability and immediately relayed the detailed directions to your location to the large spaceship, and asked the commander to come and rescue you. The commander of the spaceship responded that, unfortunately, they too had been hit by the burst of radiation that had also spun and tumbled their spaceship about and disabled their gyroscope and navigation system, but their propulsion system was still intact. You thought to yourself that you were extremely lucky that you had kept such good notes of the directions you traveled so that all the commander of the spaceship would have to do is send a rescue reconnaissance vehicle out of the same exit port of the spaceship and have it travel the same distances in the exact same directions you had traveled, and they would locate your position in space exactly. But then you realized at about the same time as did the commander of the spaceship that he needed to know in what direction the exit port of his spaceship was facing when you left the spaceship, now that the orientation of his spaceship had changed many times during the burst of interstellar radiation. He realized that he needed more than 3 dimensions to locate a point in space. He needed to know which direction on his spaceship was 'up'; i.e., orientation, when your personal reconnaissance vehicle left the spaceship. He then had to place his spaceship in the exact same orientation to be able to rescue you. His spaceship had been spun and tumbled about in many different directions and combinations of up/down, left/right, and forward/backward. Since no objects were visible and there was no gravity, and his gyroscope and navigation system were disabled, there was no frame of reference in which he could determine which way was 'up', let alone position his spaceship in the same forward direction as it had been when your reconnaissance vehicle exited the spaceship."

"There was no way," said the space dimension teacher, "that the spaceship commander could locate your position in space since he needed a $4^{\text {th }}$ space dimension to provide a frame of reference, and we call that $4^{\text {th }}$ space dimension, orientation. We take this $4^{\text {th }}$ space dimension for granted in our everyday lives because we live on an earth with gravity that provides us an automatic frame of reference and an automatic orientation in the 'up' direction. However," he says, "the story ends happily in that the commander of the spaceship was able to repair his damaged systems, and by reviewing the computer tapes of all his damaged systems, he was able to re-establish his orientation as it was when you exited his spaceship, retrace your travel and rescue you.” This little story should emphasize the point that you need 4 dimensions to locate a point in space, and orientation is that $4^{\text {th }}$ dimension. 


\section{Submit or recommend next manuscript to SCIRP and we will provide best service for you:}

Accepting pre-submission inquiries through Email, Facebook, Linkedin, Twitter, etc A wide selection of journals (inclusive of 9 subjects, more than 200 journals)

Providing a 24-hour high-quality service

User-friendly online submission system

Fair and swift peer-review system

Efficient typesetting and proofreading procedure

Display of the result of downloads and visits, as well as the number of cited articles

Maximum dissemination of your research work

Submit your manuscript at: http://papersubmission.scirp.org/ 